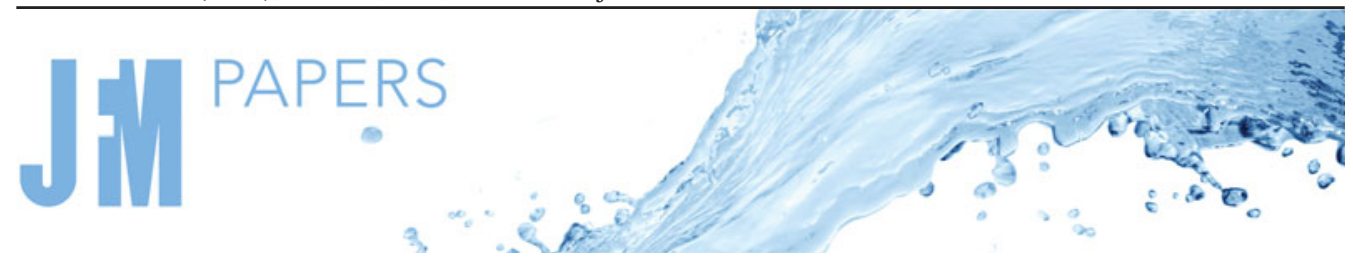

\title{
Dynamics of small heavy particles in homogeneous turbulence: a Lagrangian experimental study
}

\author{
$\operatorname{Tim}_{\text {Berk }^{1,2}}$ and Filippo Coletti ${ }^{1,2, \dagger}$ \\ ${ }^{1}$ St. Anthony Falls Laboratory, University of Minnesota, Minneapolis, MN 55414, USA \\ ${ }^{2}$ Department of Aerospace Engineering and Mechanics, University of Minnesota, Minneapolis, \\ MN 55455, USA
}

(Received 14 December 2020; revised 15 March 2021; accepted 24 March 2021)

We investigate the behaviour of microscopic heavy particles settling in homogeneous air turbulence. The regimes are relevant to the airborne transport of dust and droplets: the Taylor-microscale Reynolds number is $R e_{\lambda}=289-462$, the Kolmogorov-scale Stokes number is $S t=1.2-13$ and the Kolmogorov acceleration is comparable to the gravitational acceleration (i.e. the Froude number $F r=O(1)$ ). We use high-speed laser imaging to track the particles and simultaneously characterize the air velocity field, resolving all relevant spatio-temporal scales. The role of the flow sampled by the particles is spotlighted. In the present range of parameters, the particle settling velocity is enhanced proportionally to the velocity scale of the turbulence. Both gravity and inertia reduce the velocity fluctuations of the particles compared to the fluid; while they have competing effect on the particle acceleration, through the crossing trajectories and inertial filtering mechanisms, respectively. The preferential sampling of high-strain/low-vorticity regions is measurable, but its impact on the global statistics is moderate. The inertial particles have large relative velocity at small separations, which increases their pair dispersion; however, gravity offsets this effect by causing them to experience fluid velocities that decorrelate faster in time compared to tracers. Based on the observations, we derive an analytical model to predict the particle velocity and acceleration variances for arbitrary $S t, F r$ and $R e_{\lambda}$. This agrees well with the present observations and previous simulations and captures the respective effects of inertia and gravity, both of which play crucial roles in the transport.

Key words: particle/fluid flow, homogeneous turbulence

\footnotetext{
$\dagger$ Present address: Department of Mechanical and Process Engineering, ETH Zurich, Switzerland. Email address for correspondence: fcoletti@ethz.ch
}

(C) The Author(s), 2021. Published by Cambridge University Press. This is an Open Access article, distributed under the terms of the Creative Commons Attribution licence (http://creativecommons.org/ licenses/by/4.0/), which permits unrestricted re-use, distribution, and reproduction in any medium, provided the original work is properly cited. 


\section{T. Berk and F. Coletti}

\section{Introduction}

\subsection{Objective}

The objective of the present study is to explore the effects of inertia and gravity on the motion of heavy particles in homogeneous turbulence. Given its relevance to countless aspects of natural, industrial and medical settings, the topic has been studied in depth and a large body of literature is summarized by excellent reviews (Balachandar \& Eaton 2010; Gustavsson \& Mehlig 2016). Reaching a predictive understanding of the transport of small airborne particles is even more crucial in the current pandemic (Mittal, Ni \& Seo 2020). Still, solid evidence concerning important aspects of the particle transport (e.g. fall speed, velocity fluctuations, acceleration and dispersion) has remained elusive. The reasons include the difficulty of carrying out detailed measurements resolving all important spatio-temporal scales, the stringent hypotheses of theoretical and numerical models and the scarcity of one-to-one comparisons between computations and experiments. In particular, it is noteworthy that the majority of numerical studies have focused on the zero-gravity case. If this allows us to isolate the effect of inertia, it also impedes the direct validation in the laboratory. Indeed, several behaviours of heavy particles in turbulence were theorized and simulated for decades, e.g. the oversampling of regions of high strain and downward velocity fluctuations (Maxey 1987; Squires \& Eaton 1991b; Wang \& Maxey 1993); nevertheless, they were only recently demonstrated and quantified by experiments (Petersen, Baker \& Coletti 2019).

Here, we consider the case of turbulent air laden with solid particles much smaller than the Kolmogorov scale $\eta$. We focus on the range of Stokes number (based on the Kolmogorov time scale) $S t \approx 1-13$, while the acceleration scale of the turbulence is of the same order as the gravitational acceleration. These conditions are especially relevant to the transport of dust and droplets in the atmosphere. We perform time-resolved imaging of both the fluid and the particle motion, resolving virtually all scales at play. The measurements of particle velocity, acceleration and relative motion highlight the influence of the turbulence and the competing effects of inertia and gravity, demonstrating how in the present regime both effects are crucial for the fate and transport of the dispersed phase. The analysis culminates with an analytical model that builds on the classic framework put forward by Csanady (1963) to estimate the motion of the particles from the properties of the fluid they sample. The model is shown to agree well with the present observations, as well as with data in the literature. The rest of the paper is organized as follows: in $\S 1.2$ we summarize the theoretical background and key existing results relevant to the present study; in $\S 2$ we describe the experimental apparatus, and the measurement and processing procedure; in $\S 3$ we present and discuss the data; in $\S 4$ we introduce the analytical model and compare it with the observations, before drawing conclusions in $\S 5$.

\subsection{Background}

We briefly review some fundamental relations and concepts that will help interpret and model the behaviour of the dispersed phase. We consider particles much denser than the fluid and sufficiently small compared to any flow scale. We indicate properties of the particles, fluid and fluid at the particle location with subscripts ' $p$ ', ' $f$ ' and ' $f p$ ', respectively. If the particle Reynolds number $\operatorname{Re}_{p}$ (based on the particle diameter $d_{p}$ and a slip velocity from the fluid $u_{s}$ ) is sufficiently small, drag and gravity are the only forces usually retained in the equation of motion (Maxey \& Riley 1983)

$$
\boldsymbol{a}_{\boldsymbol{p}}=\frac{\boldsymbol{u}_{f p}-\boldsymbol{u}_{p}}{\tau_{p}}-g \hat{\boldsymbol{e}}_{\boldsymbol{y}} .
$$




\section{Dynamics of small heavy particles in homogeneous turbulence}

Here $u_{p}$ is the particle velocity, $a_{p}=\mathrm{d} u_{p} / \mathrm{d} t$ is the particle acceleration, $u_{f p}$ is the fluid velocity at the particle location (which we will refer to as the sampled-fluid velocity), $\tau_{p}$ is the particle response time and $g$ is the gravitational acceleration along the unit vector $\hat{\boldsymbol{e}}_{\boldsymbol{y}}$. We normalize using the Kolmogorov scales for time, velocity and acceleration: $\tau_{\eta}=(\nu / \varepsilon)^{1 / 2}, u_{\eta}=(\nu \varepsilon)^{1 / 4}$ and $a_{\eta}=u_{\eta} / \tau_{\eta}=\left(\varepsilon^{3} / \nu\right)^{1 / 4}$, respectively, where $v$ is the kinematic viscosity and $\varepsilon$ is the dissipation rate. This yields

$$
\frac{a_{\boldsymbol{p}}}{a_{\eta}}=S t^{-1}\left(\frac{\boldsymbol{u}_{f p}}{u_{\eta}}-\frac{\boldsymbol{u}_{\boldsymbol{p}}}{u_{\eta}}\right)-F r^{-1} \hat{\boldsymbol{e}}_{\boldsymbol{y}} .
$$

The Stokes number $S t=\tau_{p} / \tau_{\eta}$ and the Froude number $F r=a_{\eta} / g$ can be combined in the settling parameter $S v=S t / F r=\left(\tau_{p} g\right) / u_{\eta}$. We define the slip velocity as $\boldsymbol{u}_{\boldsymbol{s}}=\boldsymbol{u}_{\boldsymbol{p}}$ $\boldsymbol{u}_{f p}$, i.e. the particle velocity relative to the surrounding flow. (The opposite sign convention is also used in the literature, defining the slip velocity as the fluid velocity seen by the particle.) Denoting averaged quantities with angled brackets and fluctuating ones with a prime, the Reynolds decomposition reads

$$
a_{p}=\left\langle a_{p}\right\rangle+a_{p}{ }^{\prime}=\tau_{p}^{-1}\left(\left\langle u_{f p}\right\rangle+u_{f p}{ }^{\prime}-\left\langle u_{p}\right\rangle-u_{p}{ }^{\prime}\right)-g \hat{\boldsymbol{e}}_{y} .
$$

Averaging yields

$$
\left\langle\boldsymbol{u}_{\boldsymbol{p}}\right\rangle-\left\langle\boldsymbol{u}_{f p}\right\rangle=\left\langle\boldsymbol{u}_{s}\right\rangle=-\tau_{p}\left\langle\boldsymbol{a}_{\boldsymbol{p}}\right\rangle-\tau_{p} g \hat{\boldsymbol{e}}_{\boldsymbol{y}}
$$

and

$$
\boldsymbol{a}_{p}^{\prime}=\tau_{p}^{-1}\left(\boldsymbol{u}_{f p}^{\prime}-\boldsymbol{u}_{p}^{\prime}\right)=-\tau_{p}^{-1} \boldsymbol{u}_{s}^{\prime} .
$$

In equilibrium conditions $\left(\left\langle\boldsymbol{a}_{\boldsymbol{p}}\right\rangle=0\right)$ and still fluid $\left(\left\langle\boldsymbol{u}_{f p}\right\rangle=0\right)$ the particles settle at a terminal velocity $\left\langle u_{p, y}\right\rangle=\left\langle u_{s, y}\right\rangle=-\tau_{p} g$. Turbulence can either increase or decrease the fall speed through different mechanisms (Nielsen 1993; Good, Gerashchenko \& Warhaft 2012). Perhaps the best known among those is preferential sweeping, by which inertial particles oversample downward sides of turbulent eddies, leading to a net increase in settling velocity, especially up to $S t=O(1)$ (Maxey 1987; Wang \& Maxey 1993). Consistently with this view, Good et al. (2014) probed the parameter space and found that settling enhancement was maximum for $S t=O(1)$ and $S v=O(1)$. From (1.4), the modification of the mean vertical velocity of the particles can be expressed as $\Delta u_{y}=$ $\left\langle u_{p, y}\right\rangle+\tau_{p} g=\left\langle u_{p, y}\right\rangle-\left\langle u_{s, y}\right\rangle=\left\langle u_{f p, y}\right\rangle$ (negative for settling enhancement). That is, the settling rate modification is determined by the vertical fluid velocity sampled by the particles. It is debated which velocity scale governs the phenomenon, with various studies indicating settling enhancement proportional with the root-mean-square (r.m.s.) of the fluid velocity fluctuations $u_{r m s} \equiv\left\langle\left(u_{f}^{\prime}\right)^{2}\right\rangle^{1 / 2}$ : typically $\Delta u_{y} \approx-0.2 u_{r m s}$ (Aliseda et al. 2002; Yang \& Shy 2005; Huck et al. 2018). This enhancement was recently associated with a version of the sweep-stick mechanism in which particles oversample regions of small Lagrangian acceleration (Falkinhoff et al. 2020). Recently Momenifar \& Bragg (2020) showed by theoretical arguments and numerical simulations that the enhancement is in fact governed by a range of velocity scales, whose width increases with St. These effects lead to a settling enhancement scaling with $u_{r m s}$ for $S t=O(1)$ and $S v=O(1)$, but dependent on particle inertia outside this range as observed by Rosa \& Pozorski (2017) and Tom \& Bragg (2019). We note that, in the present study, we investigate the range in which settling enhancement scales with $u_{r m s}$, as evidenced in $\S 3.1$. 


\section{T. Berk and F. Coletti}

Averaging over the square of (1.5) leads to

$$
\left\langle\left(\boldsymbol{a}_{\boldsymbol{p}}\right)^{2}\right\rangle=\tau_{p}{ }^{-2}\left(\left\langle\left(\boldsymbol{u}_{f p}{ }^{\prime}\right)^{2}\right\rangle+\left\langle\left(\boldsymbol{u}_{\boldsymbol{p}}{ }^{\prime}\right)^{2}\right\rangle-2\left\langle\boldsymbol{u}_{f p}{ }^{\prime} \boldsymbol{u}_{\boldsymbol{p}}{ }^{\prime}\right\rangle\right)=\tau_{p}{ }^{-2}\left\langle\left(\boldsymbol{u}_{\boldsymbol{s}}\right)^{2}\right\rangle,
$$

which relates the particle velocity and acceleration variance to the sampled-fluid velocity and slip velocity variance. Of particular relevance to such a relation is the framework developed by Csanady (1963) (building on earlier work by Tchen 1947; Hinze 1975), who proposed a link between the Lagrangian spectrum of the particle velocity $\left(E_{p}\right)$ and the one of the sampled-fluid velocity $\left(E_{f p}\right)$ through a response function $H^{2}$

$$
E_{p}(\omega)=H^{2}(\omega) E_{f p}(\omega)
$$

where $\omega$ is the angular frequency in the Lagrangian frame of reference. The velocity and acceleration variances can be obtained from the spectra as

$$
\begin{gathered}
\left\langle\left(u_{p}^{\prime}\right)^{2}\right\rangle=\frac{2}{\pi} \int_{0}^{\infty} E_{p}(\omega) \mathrm{d} \omega, \\
\left\langle\left(a_{p}^{\prime}\right)^{2}\right\rangle=\frac{2}{\pi} \int_{0}^{\infty} \omega^{2} E_{p}(\omega) \mathrm{d} \omega .
\end{gathered}
$$

The response function can be modelled from (1.1), by taking the Fourier transform of the particle velocity and sampled-fluid velocity (Csanady 1963)

$$
H^{2}(\omega)=\frac{1}{1+\left(\omega \tau_{p}\right)^{2}} .
$$

Zhang, Legendre \& Zamansky (2019) derived a more complete form of the response function including unsteady forces, which, however, are expected to be small for microscopic heavy particles. The dependence of the response function on the particle response time illustrates the particle inability to respond to fluctuations with frequencies greater than $O\left(\tau_{p}^{-1}\right)$. This behaviour, often termed inertial filtering, was clearly demonstrated in situations where gravity is absent or negligible (Ayyalasomayajula et al. 2006; Bec et al. 2006). Deutsch \& Simonin (1991) made the important distinction between the spectra of the flow and the spectra of the flow sampled by the particles. In presence of gravity, already Yudine (1959) realized the importance of the drift through turbulent eddies, with particles experiencing fast-changing flow conditions: this crossing-trajectories effect leads to faster decorrelation of the particle motion (Squires \& Eaton 1991a; Elghobashi \& Truesdell 1992; Wang \& Stock 1993). Consequently, gravitational drift enhances particle acceleration compared to zero-gravity conditions, counteracting the effect of inertial filtering (Ireland, Bragg \& Collins 2016a,b). For weakly inertial particles $(S t<1)$, preferential sampling of high-strain, low-vorticity regions may also contribute to increasing particle acceleration, as the fluid acceleration is higher in the strain-dominated regions (Bec et al. 2006).

\section{Methodology}

\subsection{Experimental apparatus}

Experiments are performed in a chamber where a region of homogeneous anisotropic air turbulence is formed by two facing jet arrays. The facility was introduced and qualified in detail in Carter et al. (2016) and Carter \& Coletti (2017, 2018); here, we only give a brief description for completeness. The chamber measures $2.4 \times 2 \times 1.1 \mathrm{~m}^{3}$ in the $x, y$ and $z$ 


$\begin{array}{ccccccccc}\mu_{\text {on }}(\mathrm{s}) & u_{r m s}\left(\mathrm{~m} \mathrm{~s}^{-1}\right) & u_{r m s, x} / u_{r m s, y} & L(\mathrm{~m}) & T_{E}(\mathrm{~s}) & T_{L}(\mathrm{~s}) & \eta(\mathrm{mm}) & \tau_{\eta}(\mathrm{ms}) & R e_{\lambda} \\ 0.1 & 0.41 & 1.24 & 0.10 & 0.22 & 0.23 & 0.31 & 6.3 & 289 \\ 3.2 & 0.73 & 1.86 & 0.14 & 0.23 & 0.23 & 0.24 & 3.6 & 462\end{array}$

Table 1 . Flow properties for the cases used in the present study. The r.m.s. velocity $u_{r m s}$, integral length scale $L$ and the Eulerian and Lagrangian integral time scales $T_{E}$ and $T_{L}$ are based on weighted geometric averages over $x$ and $y$.

directions (where $x$ is aligned with the jet axis and $y$ is in vertical direction) and has acrylic walls for optical access. Each jet array consists of 128 quasi-synthetic jets, individually operated according to a sequence proposed by Variano \& Cowen (2008). The region of homogeneous turbulence (with negligible mean flow and shear) measures approximately $0.5 \times 0.7 \times 0.4 \mathrm{~m}^{3}$. The Reynolds number can be tuned by adjusting the average firing time of the jets $\left(\mu_{o n}\right)$. In the present study we operate the jets in two modes, and the main turbulence properties for each are reported in table 1. For both cases, the region of homogeneity is substantially larger than the integral length scale of the turbulence, allowing for a natural inter-scale energy cascade without major influence of the boundary conditions.

The materials and procedure followed to investigate the inertial particle transport are similar to those in Petersen et al. (2019). The particles are fed into the chamber via a $3 \mathrm{~m}$ vertical chute connected to the top of the chamber, being released at a steady rate using an AccuRate dry material feeder. We use three sizes of soda-lime glass beads (density $\rho_{p}=2500 \mathrm{~kg} \mathrm{~m}^{-3}$ ), with mean diameters $d_{p}=32 \pm 7 \mu \mathrm{m}, 52 \pm 6 \mu \mathrm{m}$ and $96 \pm 11 \mu \mathrm{m}$. Particle response times are evaluated using the Schiller \& Naumann correction (Clift, Grace \& Weber 2005)

$$
\tau_{p}=\frac{\rho_{p} d_{p}^{2}}{18 \mu\left(1+0.15 R e_{p, 0}^{0.687}\right)},
$$

where $\mu$ is the air dynamic viscosity and $R e_{p, 0}=d_{p} \tau_{p} g / \nu$ is the particle Reynolds number based on the still-air settling velocity $\tau_{p} g$. Iterative evaluation of (2.1) yields response times of $\tau_{p}=7.4,17$ and $47 \mathrm{~ms}$ respectively. The particles are expected to approach terminal velocity over a distance of the order of $\tau_{p}^{2} g$. For the largest particles this is $2 \mathrm{~cm}$, an order of magnitude smaller than the extent of the homogeneous region traversed before reaching the measurement field of view. Table 2 reports the main non-dimensional parameters for the five experimental cases obtained combining the different particle types and turbulence forcing. We focus on $S t=O(1)$ and $S v=O(1)$, with one case of larger $S t$ and $S v$. For the particle volume fraction and mass fraction in this study (of order $10^{-4}$ and $10^{-7}$, respectively), the flow properties are not expected to be modified by the loading (Petersen et al. 2019).

\subsection{Measurement approach}

Imaging is performed in the $x-y$ symmetry plane at the centre of the region of homogeneous turbulence. The flow is seeded with 1-2 $\mu \mathrm{m}$ DEHS (di-ethyl-hexyl-sebacat) droplets which faithfully follow the flow. The flow is illuminated using a Nd:YLF single-pulse laser (Photonics, $30 \mathrm{~mJ}$ pulse $^{-1}$ ) synchronized with a VEO640 camera mounting a $200 \mathrm{~mm}$ Nikon lens. An aperture number $f^{\#}=4$ gives a thickness of the focal plane of $1.5 \mathrm{~mm}$. The active portion of the camera sensor, and therefore the size of the 


$\begin{array}{lcccccc}d_{p}(\mu \mathrm{m}) & R e_{\lambda} & S t & S v & F r & d_{p} / \eta & R e_{p, 0} \\ 32 & 289 & 1.2 & 1.5 & 0.8 & 0.10 & 0.15 \\ 32 & 462 & 2.1 & 1.1 & 1.9 & 0.13 & 0.15 \\ 52 & 289 & 2.7 & 3.4 & 0.8 & 0.16 & 0.56 \\ 52 & 462 & 4.7 & 2.5 & 1.9 & 0.21 & 0.56 \\ 96 & 462 & 13.1 & 6.9 & 1.9 & 0.42 & 3.26\end{array}$

Table 2. Relevant non-dimensional parameters for the experimental cases in the present study.

$\begin{array}{cccccc}\operatorname{Re}_{\lambda} & f_{a q}(\mathrm{~Hz}) & \tau_{\eta} f_{a q} & T_{a q} / T_{L} & \mathrm{FOV}(x \times y)\left(\mathrm{cm}^{2}\right) & \text { Resolution }(\text { pix mm} \\ & -1 & \\ 289 & 1800 & 11.3 & 38 & 11.5 \times 9.0 & 17.8 \\ 462 & 5100 & 18.4 & 44 & 5.8 \times 5.4 & 17.8\end{array}$

Table 3. Acquisition parameters for the two turbulence forcing cases; $f_{a q}$ and $T_{a q}$ represent the acquisition frequency and length of acquisition, respectively.

field of view (FOV), depends on the acquisition frequency. The latter is optimized to give a time separation between consecutive images of at most $0.1 \tau_{\eta}$, yielding the resolutions reported in table 3. For both $R e_{\lambda}$, the FOV is much smaller than the region of homogeneous turbulence. For each case we record 10 separate runs, with total duration of the recordings of around 40 integral time scales.

Data are processed using the procedure detailed in Petersen et al. (2019). Raw images are separated in particle-only images and tracer-only images, distinguishing DEHS droplets and glass beads based on brightness and size. Particle image velocimetry (PIV) is performed on the tracer-only images. We use an initial interrogation window of 64times64 pixels, refined to $32 \times 32$ pixels with $50 \%$ overlap, for a vector spacing of $0.9 \mathrm{~mm}(2.9 \eta$ and $3.7 \eta$ for the $R e_{\lambda}=289$ and 462 cases respectively) that resolves the fine scales of turbulence (Worth, Nickels \& Swaminathan 2010). Particle tracking velocimetry (PTV) is performed on the particle-only images, following the cross-correlation approach. The fluid velocity is evaluated at the particle locations using weighted linear interpolation of the four neighbouring velocity vectors. A comparison with cubic and spline interpolation shows no significant difference, but linear interpolation substantially decreases the computation time. Particle velocities and accelerations are determined from the particle position using convolution with the first and second derivatives of a Gaussian kernel, respectively. The width of the latter is chosen as $0.5 \tau_{\eta}$ and $0.4 \tau_{\eta}$ for the $\operatorname{Re}_{\lambda}=289$ and 462 cases, respectively, following the procedure established for tracers in Voth et al. (2002) and Mordant, Lévêque \& Pinton (2004) and applied to inertial particles in Gerashchenko et al. (2008), Nemes et al. (2017) and Ebrahimian, Sanders \& Ghaemi $(2019 b, a)$.

The number of samples used to calculate particle statistics ranges between $0.8 \times 10^{6}$ and $5.6 \times 10^{6}$ for the different cases. Uncertainty in the statistics is affected by both random uncertainty, due to the finite sample size, and bias uncertainty, due to systematic errors in estimating the particle centroid and the local fluid velocity. Baker \& Coletti (2021), who followed a similar time-resolved PIV/PTV approach for particle-laden turbulent boundary layers, showed that the bias uncertainty was negligible compared to the random uncertainty. Compared to their study, the present particles are much smaller with respect to the flow scales, and therefore the error associated with interpolating the fluid velocity at the particle location is correspondingly smaller. The centroid location uncertainty 


$\begin{array}{cccccccc}\left\langle u_{f p, y}\right\rangle & \left\langle u_{p, y}\right\rangle & \left\langle u_{s, y}\right\rangle & \left\langle\left(u_{f p, y}^{\prime}\right)^{2}\right\rangle & \left\langle\left(u_{p, y}^{\prime}\right)^{2}\right\rangle & \left\langle\left(u_{s, y}^{\prime}\right)^{2}\right\rangle & \left\langle\left(\mathrm{d} u_{f p, y}^{\prime} / \mathrm{d} t\right)^{2}\right\rangle & \left\langle\left(a_{p, y}^{\prime}\right)^{2}\right\rangle \\ 1.4 \% & 0.8 \% & 0.3 \% & 1.3 \% & 1.2 \% & 0.3 \% & 0.3 \% & 0.3 \%\end{array}$

Table 4. Random errors of the velocity and acceleration statistics based on the standard deviation of the last $20 \%$ of data for the case with the lowest number of samples.
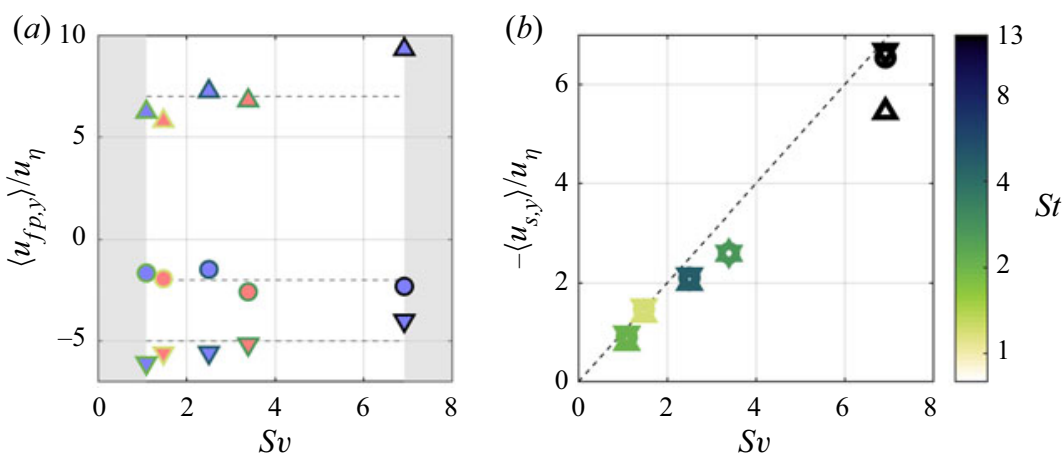

Figure 1. Mean vertical velocity of the sampled flow $(a)$ and mean vertical slip velocity $(b)$. Symbols represent the mean over the upward- and downward-moving subsets (upward- and downward-pointing triangles, respectively) as well as the ensemble mean (circles). Dashed lines in $(a)$ represent $\left\langle u_{f p, y}\right\rangle / u_{\eta}=C$, with $C$ defined in the text. The dashed line in $(b)$ represents $\left\langle u_{s, y}\right\rangle=-\tau_{p} g$. Blue and red shadings in $(a)$ represent the low (red) and high (blue) $R e_{\lambda}$ cases. The colour bar here and in subsequent figures indicates the colouring of symbols with respect to $S t$ (in some figures with respect to $S v$ ).

(investigated for these same particles in Petersen et al. 2019) is an order of magnitude smaller than the typical particle displacement as in Baker \& Coletti (2021), and therefore its impact is also deemed negligible compared to the random uncertainty. The latter is estimated based on the standard deviation of the last $20 \%$ of data (Ebrahimian et al. $2019 a$ ), and is reported in table 4 for the main quantities.

\section{Results}

\subsection{Velocity}

We begin by considering the statistics of the sampled fluid, focusing on the vertical component $u_{f p, y}$ which is most relevant to the settling process. Figure 1(a) shows the mean vertical sampled-fluid velocity for all particles, as well as the velocity conditioned on upward-/downward-moving particles (i.e. particles with positive/negative $u_{f p, y}$ ). The results are approximately independent of $S v$ and consistent with the scaling $\left\langle u_{f p, y}\right\rangle / u_{\eta}=$ $C$, where the constant $C$ is the mean for the respective sets of particles. Given the expected dependence of the vertical velocities with $S t$ and $S v$, here and in the following figure we shade the area outside of the investigated range to emphasize that the trends should not be extrapolated. According to previous studies mentioned in $\S 1$, we expect $\left\langle u_{f p, y}\right\rangle \approx-0.2 u_{r m s}$, which over the present range of $R e_{\lambda}$ implies $\left\langle u_{f p, y}\right\rangle \approx-2 u_{\eta}$. Indeed, for the unconditional average over all particles we find $C \approx-2$. The results for the downward-moving and upward-moving subsets are consistent with $C \approx-5$ and $C \approx 7$, respectively. Thus, the upward-/downward-moving particles sample fluid regions with relatively large upward/downward velocity fluctuations, and these are of similar 


\section{T. Berk and F. Coletti}
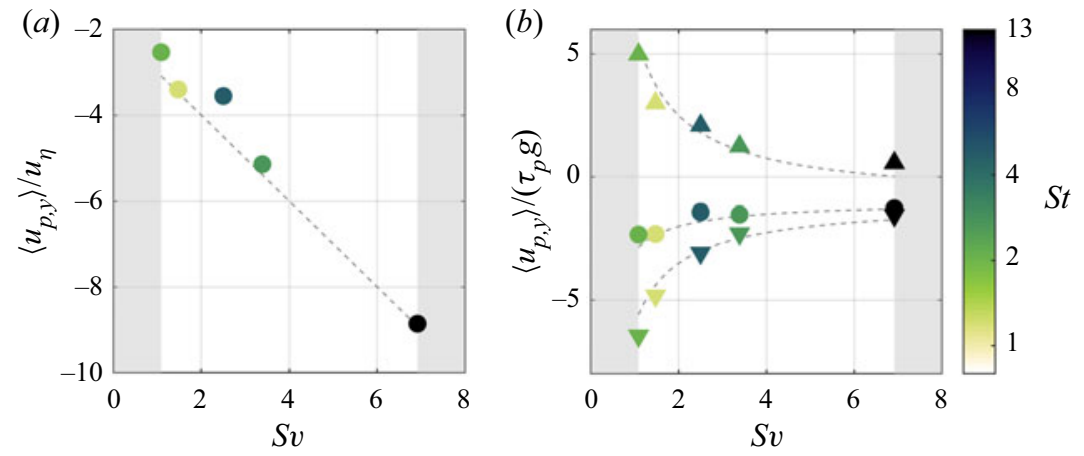

Figure 2. Mean vertical particle velocity, normalized by the Kolmogorov velocity $(a)$ and by the still-air settling velocity $\tau_{p} g(b)$. Symbols represent the mean over the upward- and downward-moving subsets (upwardand downward-pointing triangles, respectively) as well as the ensemble mean (circles). The dashed line in (a) represents $\left\langle u_{s, y}\right\rangle / u_{\eta}=-S v$, with $C$ defined in the text. Dashed lines in $(b)$ represent $\left\langle u_{p, y}\right\rangle /\left(\tau_{p} g\right)=$ $C S v^{-1}-1$.
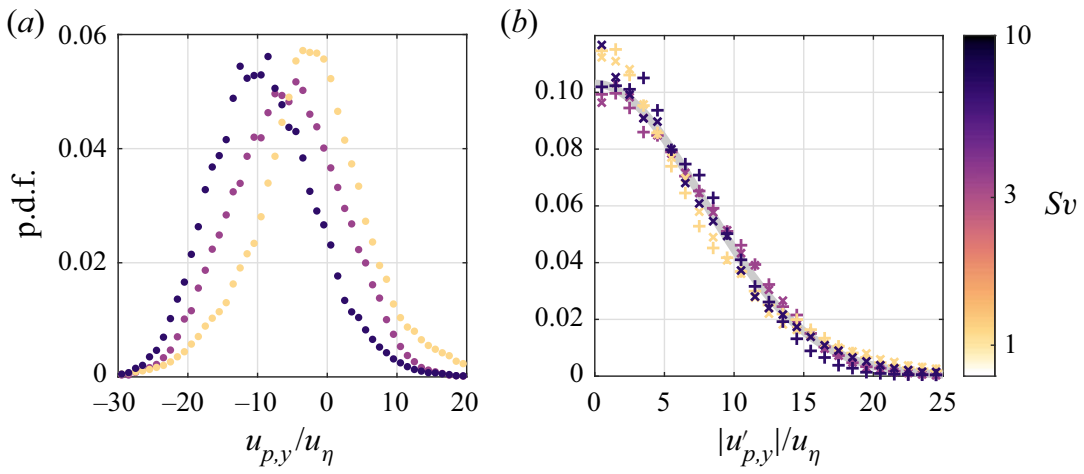

Figure 3. The p.d.f.s of the vertical particle velocity for select cases $(a)$. The p.d.f.s of the absolute value of the fluctuating part of the vertical particle velocity $(b)$. Here, ' $x$ ' denotes upward-moving particles while ' + ' denotes downward-moving particles. The solid grey line indicates a Gaussian distribution.

magnitude for both subsets. Therefore, the net settling enhancement stems from the downward particles being more numerous, not from their association with stronger downward events.

Figure $1(b)$ shows the normalized slip velocity, the dashed line indicating $\left\langle u_{s, y}\right\rangle / u_{\eta}=$ $-S v$, or $\left\langle u_{s, y}\right\rangle=-\tau_{p} g$, as theorized by Wang \& Maxey (1993). This corresponds well to the measured data, except for the upward-moving particles of largest $S v$, which have a significantly smaller slip velocity. This is likely related to the assumption $\left\langle a_{p}\right\rangle=0$. The latter is strictly valid only for the ensemble of all particles, and not necessarily for specific subsets. In absence of this assumption, (1.4) predicts a smaller slip velocity for mean downward particle acceleration.

We then consider the particle vertical mean velocity $\left\langle u_{p, y}\right\rangle$. Since in the considered regimes we have approximately $\left\langle u_{f p, y}\right\rangle / u_{\eta}=C$ and $\left\langle u_{s, y}\right\rangle / u_{\eta}=-S v$, we expect $\left\langle u_{p, y}\right\rangle / u_{\eta}=C-S v$. Figure 2(a) supports this scaling for the ensemble of all particles. Similarly, normalizing by the still-air terminal velocity leads to $\left\langle u_{p, y}\right\rangle /\left(\tau_{p} g\right)=C / S v-1$. This is confirmed in figure $2(b)$, where the scaling is shown to hold also for upward and downward-moving particles (with the respective values of the constant). We again stress 


\section{Dynamics of small heavy particles in homogeneous turbulence}
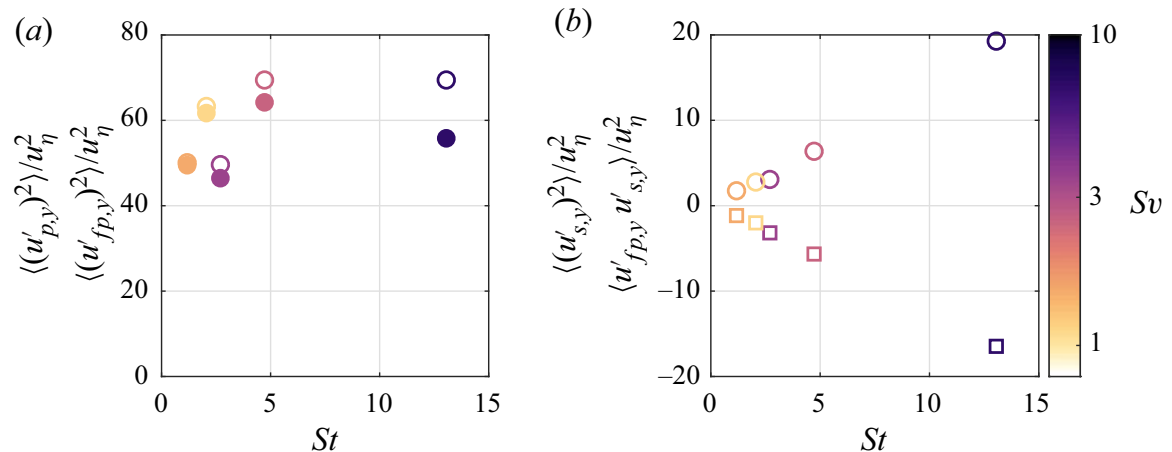

Figure 4. Variance of vertical particle velocity (solid symbols) and sampled-fluid velocity (open symbols) ( $a$ ). Variance of vertical slip velocity (circles) and covariance of the vertical slip velocity and sampled-fluid velocity (squares) $(b)$.

that this cannot be extrapolated ad libitum: in the limit of both vanishing and infinite particle inertia, we expect $\left\langle u_{p, y}\right\rangle /\left(\tau_{p} g\right)=-1$.

The probability density function (p.d.f.) of the normalized vertical particle velocity, $u_{p, y} / u_{\eta}$, is shown in figure 3(a) for three selected cases, the other cases sharing the same trends. For increasing $S v$, the distributions shifts to more negative values, as expected, and the distributions become positively skewed. This is in contrast with the findings of Baker et al. (2017), who used point-particle simulations. The disagreement for the heavier particles is not surprising, as the assumptions behind such simulations become questionable with increasing $R e_{p}$. Figure $3(b)$ reports the p.d.f. of the absolute value of the particle velocity fluctuations, $\left|u_{p, y}^{\prime}\right| / u_{\eta}$, distinguishing between upward- and downward-moving particles. We observe no difference between both subsets, and therefore in the following we do not consider them separately. All $S v$ cases collapse well on a Gaussian distribution when normalized by the Kolmogorov velocity. This confirms the dominant role of the fluid fluctuations in determining the particle velocity fluctuations, for a wide range of response times and fall speeds.

We then investigate the scaling of the particle velocity fluctuations by considering the variance of $\boldsymbol{u}_{p}{ }^{\prime}=\boldsymbol{u}_{f p}{ }^{\prime}+\boldsymbol{u}_{s}{ }^{\prime}$

$$
\left\langle\left(\boldsymbol{u}_{\boldsymbol{p}}{ }^{\prime}\right)^{2}\right\rangle=\left\langle\left(\boldsymbol{u}_{f p}{ }^{\prime}\right)^{2}\right\rangle+\left\langle\left(\boldsymbol{u}_{\boldsymbol{s}}{ }^{\prime}\right)^{2}\right\rangle+2\left\langle\boldsymbol{u}_{f p}{ }^{\prime} \boldsymbol{u}_{\boldsymbol{s}}{ }^{\prime}\right\rangle .
$$

Figure 4 displays the vertical components of the four terms in (3.1) for the various cases, normalized by Kolmogorov scaling. The horizontal components, not shown, behave similarly. The particle velocity variance $\left\langle\left(u_{p, y}^{\prime}\right)^{2}\right\rangle$ is smaller than but comparable to the variance of the sampled-fluid velocity $\left\langle\left(u_{f p, y}^{\prime}\right)^{2}\right\rangle$ (figure $4 a$ ), as also reported by Ireland et al. (2016a) in zero-gravity simulations. This confirms that, in the present range of parameters, the fluctuating energy of the particles is driven by the turbulent kinetic energy. Figure $4(b)$ indicates that the normalized slip velocity variance $\left\langle\left(u_{s, y}^{\prime}\right)^{2}\right\rangle / u_{\eta}^{2}$ varies linearly with $S t$. This is consistent with the scaling $u_{S} / u_{\eta} \propto S t^{1 / 2}$ in Balachandar (2009), derived for the present range $\left(\tau_{\eta}<\tau_{p}<T_{L}\right)$ but in the absence of gravity. The covariance $\left\langle u_{f p, y}^{\prime} u_{s, y}^{\prime}\right\rangle$ also varies linearly with $S t$ and approximately equals $-\left\langle\left(u_{s, y}^{\prime}\right)^{2}\right\rangle$. Therefore, from (3.1) we have $\left\langle\left(u_{p, y}^{\prime}\right)^{2}\right\rangle \approx\left\langle\left(u_{f p, y}^{\prime}\right)^{2}\right\rangle-\left\langle\left(u_{s, y}^{\prime}\right)^{2}\right\rangle$. As $\left\langle\left(u_{s, y}^{\prime}\right)^{2}\right\rangle$ grows with $S t$, we retrieve the influence of inertial filtering: heavier particles exhibit weaker velocity fluctuations with respect to the sampled-fluid fluctuations. 


\section{T. Berk and F. Coletti}

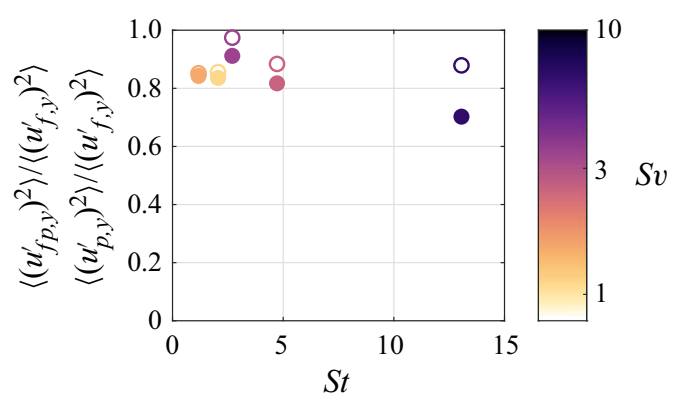

Figure 5. Variances of the vertical sampled-fluid velocity (open symbols) and vertical particle velocity (solid symbols), normalized by the fluid velocity variance.

We conclude this section by comparing the sampled-fluid and particle velocity variances against the fluid velocity variance, again focusing on the vertical components (figure 5). This allows us to quantitatively compare the fluctuating energy of the dispersed and carrier phase. The zero-gravity simulations of Ireland et al. (2016a) indicated that the particle velocity fluctuations can exceed the fluid fluctuations for $S t<1$, due to preferential sampling of energetic flow regions. In the present case, on the other hand, both inertia and gravity concur to reduce the particle fluctuating energy below the turbulent kinetic energy of the fluid, in agreement with the algebraic model of Wang \& Stock (1993) and the results of Good et al. (2014) in similar ranges of St and Sv. In particular, figure 5 shows that the fluctuating energy of the fluid sampled by the particles is somewhat lower than (although comparable to) the unconditional turbulent kinetic energy. This implies that, as far as this observable is concerned, the effect of preferential sampling is relatively weak. The latter is indeed offset by gravitational drift, which reduces the particle ability to follow energetic fluid structures, hence $\left\langle\left(u_{f p, y}^{\prime}\right)^{2}\right\rangle<\left\langle\left(u_{f, y}^{\prime}\right)^{2}\right\rangle$. This picture will be confirmed later, when analysing the fast decorrelation of the sampled-fluid velocity (see $\S 4.2)$. Inertial filtering further reduces $\left\langle\left(u_{p, y}^{\prime}\right)^{2}\right\rangle$ with respect to $\left\langle\left(u_{f p, y}^{\prime}\right)^{2}\right\rangle$, and therefore the former is $10-30 \%$ lower than $\left\langle\left(u_{f, y}^{\prime}\right)^{2}\right\rangle$.

\subsection{Acceleration}

We present results for the vertical components of the particle acceleration, $a_{p, y}$, the horizontal components behaving similarly. In addition, we consider the temporal derivative of the sampled-fluid velocity, $\mathrm{d} u_{f p, y} / \mathrm{d} t$. The p.d.f.s of the latter are presented in figure $6(a)$, showing similar distributions for the cases with the same $R e_{\lambda}$. Indeed, for vanishing inertia $\mathrm{d} u_{f p, y} / \mathrm{d} t$ equals the Eulerian acceleration which, in homogeneous turbulence, is a function of $R e_{\lambda}$ only (Hill 2002; Sawford et al. 2003). For a given $R e_{\lambda}$, the distributions of $\mathrm{d} u_{f p, y} / \mathrm{d} t$ become wider with $S v$, in agreement with the simulations of Ireland et al. (2016b). This is a manifestation of the crossing-trajectories effect: for higher settling rate, the particles experience rapid changes of the sampled-fluid environment. The distributions of $a_{p, y}$ (figure $6 b$ ) are much narrower than the corresponding distributions of $\mathrm{d} u_{f p, y} / \mathrm{d} t$, due to inertial filtering. This effect becomes stronger with larger particle inertia, which in the present case implies an increase of both $S t$ and $S v$.

The variances of $\mathrm{d} u_{f p, y} / \mathrm{d} t$ and $a_{p, y}$ are quantified in figure 7. As observed above, the variance of $\mathrm{d} u_{f p, y} / \mathrm{d} t$ is dominated by $R e_{\lambda}$ and increases with $S v$. For tracers in homogeneous isotropic turbulence, the normalized acceleration variance of the fluid can be approximated as $a_{0} \equiv\left\langle a_{f}^{2}\right\rangle / a_{\eta}^{2}=5 /\left(1+100 R e_{\lambda}^{-1}\right)$ (Sawford et al. 2003), hence for 

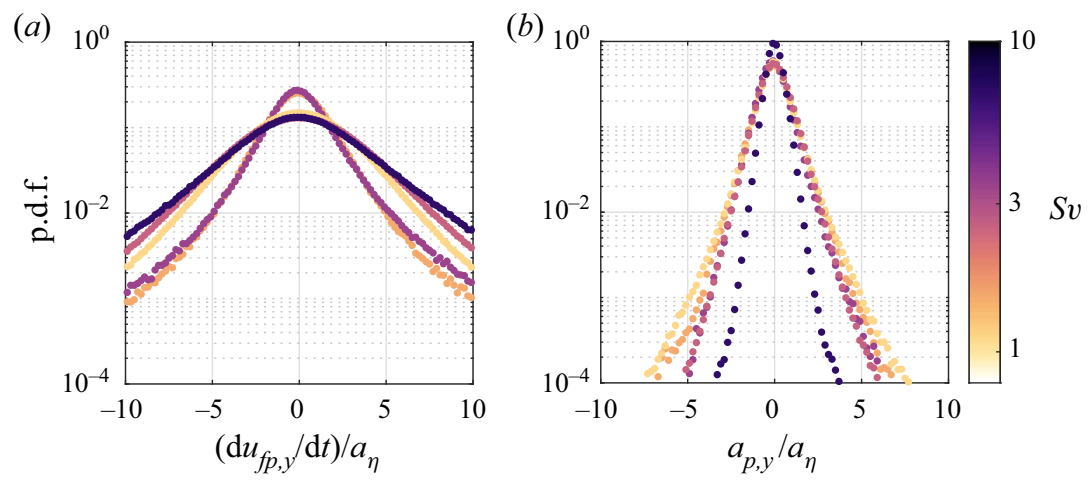

Figure 6. The p.d.f.s of the vertical components of the temporal derivative of the sampled-fluid velocity $(a)$ and the particle acceleration $(b)$.
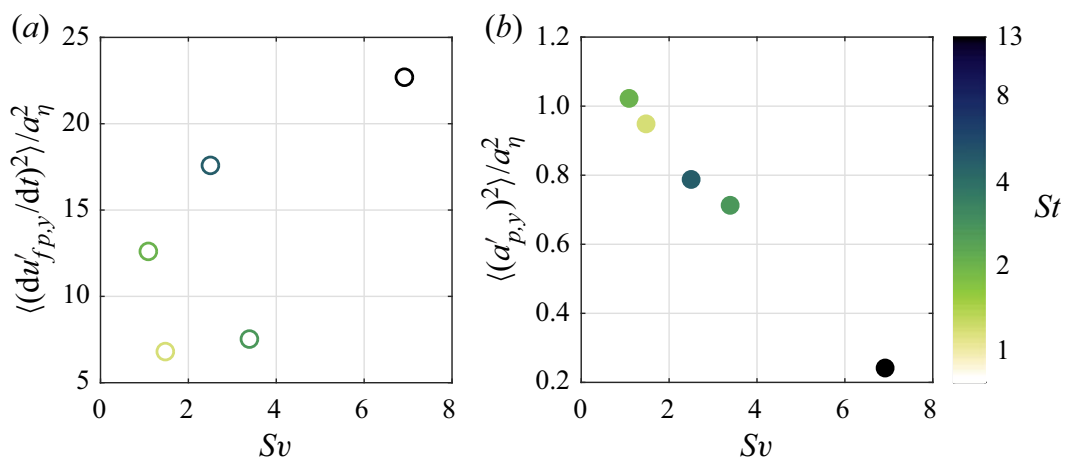

Figure 7. Variances of the vertical components of the temporal derivative of the sampled-fluid velocity $(a)$ and the particle acceleration $(b)$.

$R e_{\lambda}=289$ and 462 we expect $a_{0}=3.6$ and 4.0, respectively. Ireland et al. (2016b) showed a monotonic increase of $\left\langle\left(\mathrm{d} u_{f p, y}^{\prime} / \mathrm{d} t\right)^{2}\right\rangle / u_{\eta}^{2}$ roughly with $S v^{3 / 2}$, independent of $R e_{\lambda}$. In the present study, the variance of $\mathrm{d} u_{f p, y} / \mathrm{d} t$ is larger than for tracers, but not as large as in Ireland $e t a l$. (2016b) and still dependent on $R e_{\lambda}$. The difference with Ireland et al. (2016b) is reflected in the Froude number: in their study $F r=0.052$, while here $F r=0.8$ and 1.9, for $R e_{\lambda}=289$ and 462 respectively. For $F r=O(1)$, gravitational and Kolmogorov acceleration are of the same order of magnitude, i.e. fluid turbulence and gravity are expected to have comparable influences. The variance of $a_{p, y}$ (figure $7 b$ ) decreases approximately linearly with $S v$ in the present range. It is significantly smaller than the variance of $\mathrm{d} u_{f p, y} / \mathrm{d} t$ and also smaller than $a_{0}$, due to inertial filtering.

\subsection{Preferential sampling}

To quantify the extent and influence of preferential sampling, we discriminate between rotation-dominated and strain-dominated fluid regions using the second invariant of the velocity gradient tensor (Hunt, Wray \& Moin 1988)

$$
Q=\omega^{2} / 4-s^{2} / 2,
$$




\section{T. Berk and F. Coletti}

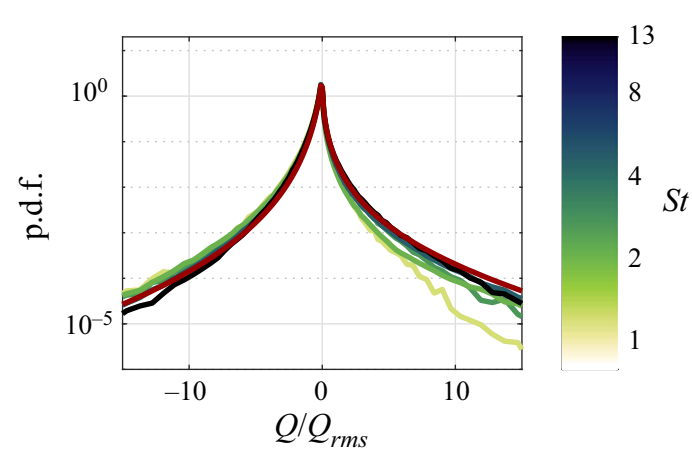

Figure 8. The p.d.f.s of the particle-sampled $Q$, defined in (3.2). The red line indicates ensemble averaged (i.e. unconditional) $Q$ (similar for both Reynolds numbers).

where $\omega^{2}=2 \operatorname{tr}\left(\boldsymbol{\Omega}^{2}\right)$ is the enstrophy, $s^{2}=\operatorname{tr}\left(\boldsymbol{S}^{2}\right)$ is the squared strain rate and $\boldsymbol{S}$ and $\boldsymbol{\Omega}$ are the symmetric and anti-symmetric part of the velocity gradient tensor, respectively. Due to the planar nature of the measurements, we can only consider the in-plane components of the velocity gradient tensor, which are not sufficient to fully describe the flow topology (Perry \& Chong 1994). Still, especially in homogeneous turbulence, the in-plane part of the tensor provides important physical insight into the properties of high-enstrophy and high-strain structures (Cardesa et al. 2013) and captures the fundamental small-scale features (Fiscaletti, Ganapathisubramani \& Elsinga 2015; Carter $\&$ Coletti 2018). Figure 8 shows the p.d.f. of $Q$ evaluated at the particle locations, compared to the unconditioned fluid. For the smaller $S t$ considered, the particle-conditioned distributions display the expected under-sampling of rotation-dominated regions $(Q>0)$, which, however, becomes progressively weaker as $S t$ increases above unity.

The effect of the small-scale features of the sampled fluid on the particle motion is depicted in figure 9, which plots the particle acceleration variance conditioned on strain rate (figure 9a) and enstrophy (figure $9 b$ ). In both cases, larger levels of small-scale turbulence activity correspond to stronger accelerations. Although the correlation with $s^{2}$ is somewhat stronger than with $\omega^{2}$, the similarity of both plots is consistent with the view that high-strain and high-enstrophy events are often concurrent (Worth \& Nickels 2011; Yeung, Donzis \& Sreenivasan 2012; Carter \& Coletti 2018). Due to inertial filtering, the impact of the sampled-fluid topology decreases steeply with $S t$. This is clearly shown in figure $9(c)$, displaying the variance of the particle acceleration conditioned on the sign of $Q$ : particles in strain-dominated regions do display larger accelerations (Bec et al. 2006), but the effect becomes unmeasurable for $S t=4.7$ and larger.

\subsection{Structure functions and pair dispersion}

In this section we consider two-particle statistics, starting with the second-order Eulerian velocity structure function $S_{2}(r)=\left\langle\left(\boldsymbol{u}^{\prime}(\boldsymbol{x})-\boldsymbol{u}^{\prime}(\boldsymbol{x}+\boldsymbol{r})\right)^{2}\right\rangle$, where $\boldsymbol{r}$ is the separation vector. For fluid tracers in homogeneous isotropic turbulence, this scales as $r^{2}$ in the dissipative range, plateaus to the fluid velocity variance at large-scale separations, and follows the scaling predicted by Kolmogorov (1941) in the inertial range

$$
\begin{array}{ccc}
S_{2 \|}(r)=C_{2}(\varepsilon r)^{2 / 3} & \text { or } & S_{2 \|} / u_{\eta}^{2}=C_{2}(r / \eta)^{2 / 3}, \\
S_{2 \perp}(r)=\frac{4}{3} C_{2}(\varepsilon r)^{2 / 3} & \text { or } & S_{2 \perp} / u_{\eta}^{2}=\frac{4}{3} C_{2}(r / \eta)^{2 / 3},
\end{array}
$$



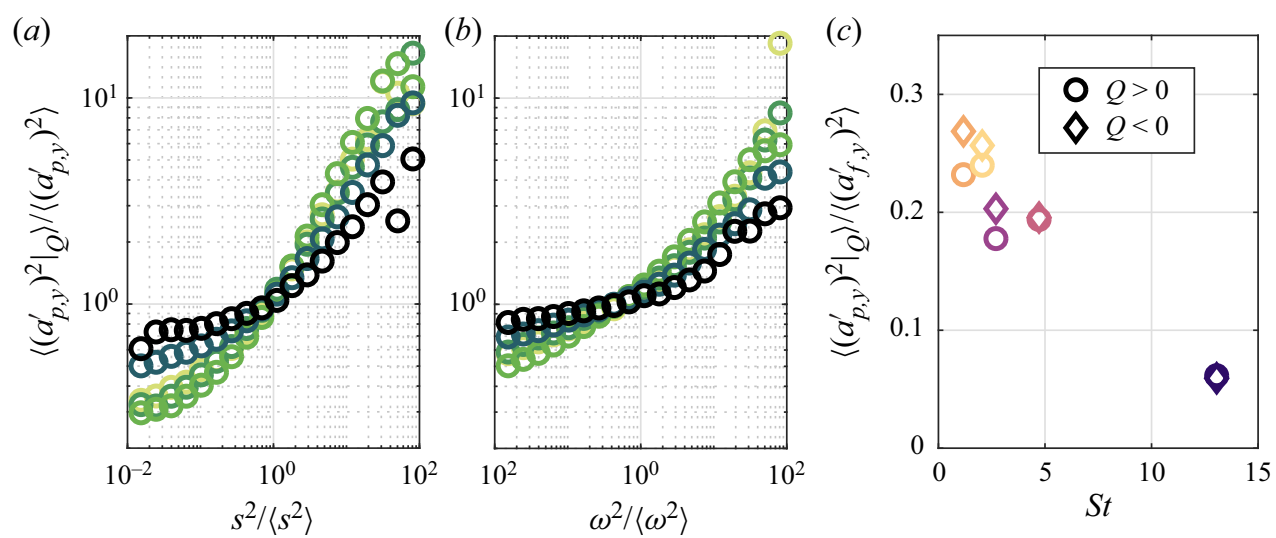

Figure 9. Vertical component of the particle acceleration variance, conditioned to $Q$. Acceleration variance conditioned to high-strain events for $Q<0(a)$ and to high-enstrophy events for $Q>0(b)$, both normalized by the variance of the ensemble. Acceleration variance averaged over $Q<0$ (diamonds) and $Q>0$ (circles), normalized by the variance of the fluid acceleration, calculated as $\left\langle\left(a_{f, y}^{\prime}\right)^{2}\right\rangle=a_{0} a_{\eta}(c)$.

where the subscripts $\|$ and $\perp$ denote velocity components longitudinal and transverse to the separation vector, respectively, and $C_{2} \approx 2$ (Saddoughi \& Veeravalli 1994). For non-tracer particles, inertia and gravity modify these trends. Simulations and experiments indicate that, when gravitational effects are negligible or absent, inertia leads to greater relative velocities between nearby particles, such that the structure function increasingly deviates from the $r^{2}$ scaling at small separations (Bec et al. 2010; Ireland et al. 2016a; Dou et al. 2018). This is attributed to the path-history effect, i.e. the particles retaining memory of their past interactions with the flow and thus approaching each other with a significant uncorrelated velocity component (see, among many others, Wilkinson \& Mehlig 2005; Fevrier, Simonin \& Squires 2005; Bragg \& Collins 2014; Fong, Amili \& Coletti 2019). With the addition of gravity, the simulations of Ireland et al. (2016b) indicated a strong reduction of relative particle velocity at all separations. They attributed this to the decorrelation of the sampled-fluid velocity along the particle trajectories (which we shall confirm later), hindering the path-history effect and in turn decreasing the relative velocities. To our best knowledge, no previous experimental observation could verify this latter point.

The longitudinal structure functions are presented in figure 10, the transverse components (not shown) showing analogous trends. Results are compared to the measured Eulerian structure function for tracers, which follows both $r^{2}$ scaling in the dissipative range and the scaling from (3.3) in the inertial range. Due to the finite laser sheet thickness $(\approx 6 \eta)$ we expect an overestimation of the relative velocity over the dissipative range (Dou et al. 2018), which, however, may not overwhelm the trend. At small separations $(r \lesssim 20 \eta)$, the structure functions of the particles deviate from tracers with increasing $S t$, confirming previous findings. In the inertial range, $S_{2 \|}(r)$ roughly follows the $r^{2 / 3}$ scaling, but the values are significantly lower than for tracers. The gap persists at large scales, consistent with the fact that the inertial particle fluctuating energy (to which the structure function asymptotes for large $r$ ) is lower compared to the fluid, see figure 5. Although the competing effects of inertia and gravity cannot be separated here, these results appear to confirm the observation of Ireland et al. (2016b) that gravity reduces the relative particle velocities at all scales. 


\section{T. Berk and F. Coletti}

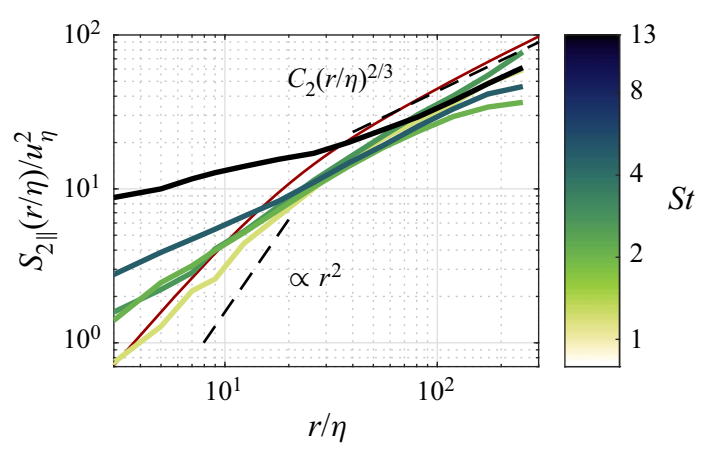

Figure 10. Longitudinal structure functions using the particle velocity. The red line indicates the (Eulerian) structure function of tracers. Dashed lines indicate $r^{2}$ scaling and the prediction by Kolmogorov (1941) for the inertial range, respectively.

We then turn to particle pair separations as a function of time, $r(t)$. In homogeneous isotropic turbulence, for tracer pairs with an initial separation $r_{0}$ in the inertial range, we expect the mean square separation to follow the ballistic scaling proposed by Batchelor (1950)

$$
\left\langle\left(r(t)-r_{0}\right)^{2}\right\rangle=\frac{11}{3} C_{2}\left(\varepsilon r_{0}\right)^{2 / 3} t^{2}, \quad \text { for } \tau_{\eta} \ll t \ll t_{B},
$$

where $t_{B}=\left(r_{0}^{2} / \varepsilon\right)^{1 / 3}$ is the characteristic time scale of an eddy of size $r_{0}$. Recently, a more general time scale $t_{0}=S_{2}\left(r_{0}\right) / \varepsilon$ has been proposed by Bitane, Homann \& Bec (2012). For $t_{B} \ll t \ll T_{L}$ (or $t_{0} \ll t \ll T_{L}$ ), the dispersion does not depend on $r_{0}$ and is expected to follow the Richardson-Obukhov scaling $\left\langle r(t)^{2}\right\rangle=g \varepsilon t^{3}$, where $g \approx 0.5$ (Salazar \& Collins 2012). For $t$ in the dissipative range, particle inertia enhances pair dispersion at small times, due to the large relative velocities at small separation (Bec et al. 2010; Gibert, Xu \& Bodenschatz 2010); while for larger $t$, the inertial filtering and path-history effects reduce pair dispersion compared to tracers (Bragg, Ireland \& Collins 2016). To the best of our knowledge, the only previous investigations on the effect of gravity on pair dispersion are the numerical studies by Chang, Malec \& Shaw (2015) and Dhariwal \& Bragg (2019), who mostly focused on bi-dispersed particles sets.

The mean square separation for our inertial particles is presented in figure 11. Due to the nature of the measurements, the results are biased by the constraint that the trajectories cannot separate more than the laser sheet thickness in $z$. One way to account for this is to consider that the right-hand side in (3.5) is the geometric average of the structure function components at $r_{0}$, i.e. $\left\langle\left(r(t)-r_{0}\right)^{2}\right\rangle=\left(S_{2 \|}\left(r_{0}\right)+2 S_{2 \perp}\left(r_{0}\right)\right) t^{2}$. Setting to zero the out-of-plane velocity, we can write the mean square separation for tracers as $\frac{7}{3} C_{2}\left(\varepsilon r_{0}\right)^{2 / 3} t^{2}$, which is plotted as a black solid line for reference. The effect of $S t$ (and indirectly of $S v$ ) is represented in figure 11(a), for trajectories with initial separation $3<r_{0} / \eta<4$. With increasing particle inertia, the mean square separation grows. Still, all curves are generally below the expectation for tracers, illustrating the competing effect of inertia and gravity. The normalized form plotted in figure 11(b) illustrates the effect of the initial separation for the case $S t=13$ : with increasing initial separations, the impact of the large relative velocities is weakened, and the curves tend to collapse on each other (which is also the case for smaller St, not shown). This confirms the strong influence of the uncorrelated motion of nearby particles, already highlighted by the structure functions. The plot also emphasizes how the particles follow the Batchelor regime up to $t=O\left(\tau_{\eta}\right)$, which corresponds as well to $t=O\left(t_{B}\right)$ and $t=O\left(t_{0}\right)$. Around $t=O\left(\tau_{\eta}\right)$ we observe a 

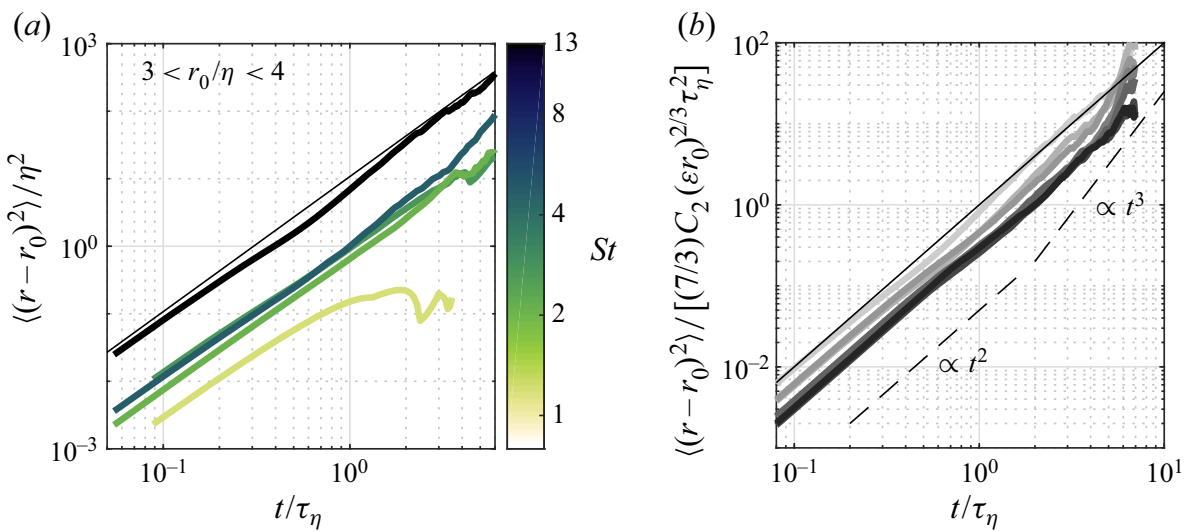

Figure 11. Mean square separation of particle pairs with initial separation $r_{0}$. Mean square separation of pairs with $3<r_{0} / \eta<4$ for all cases (a). Mean square separation of pairs with $3<r_{0} / \eta<4$ to $9<r_{0} / \eta<10$ (increasing with darker shades) for the $S t=13$ case (b). The solid thin lines indicate the scaling in (3.5).

mild slowing down of the ballistic separation, also observed for tracers by Bourgoin (2015), after which the slope increases. However, given the bias due to the shape of the illuminated volume, caution should be exerted when interpreting the behaviour for relatively long times. Here, we just note that, for particles in zero gravity, the transition out of the ballistic regime is expected to ensue at times $O\left(\tau_{p}\right)$ (Bragg et al. 2016), i.e. much later than what we observe. The difference appears to be rooted in the effect of gravity and could be associated with the time scale of eddy crossing by the falling particles, similarly to recent findings for rising bubbles (Mathai et al. 2018). Further research is warranted on this point.

\section{Modelling the velocity and acceleration variances}

Here, we leverage and expand on the framework of Csanady (1963) to obtain expressions for the particle velocity and acceleration variances. To summarize the detailed explanation that follows, we integrate the Lagrangian particle velocity spectrum, which is obtained from the sampled-fluid velocity spectrum modulated by a response function. To extend the frequency range of the spectrum, we Fourier transform the Sawford (1991) expression of the velocity autocorrelation. As this was originally derived for the unconditional fluid, we substitute in it the Lagrangian time scales associated with the sampled fluid.

\subsection{Lagrangian spectrum of particle velocity}

For frequencies in the inertial range $\pi / T_{L} \ll \omega \ll \pi / \tau_{\eta}$, the Lagrangian spectrum for the fluid flow is expected to scale as $E \propto \varepsilon \omega^{-2}$ (Tennekes \& Lumley 1972; Yeung 2001). The spectrum can also be derived from the velocity autocorrelation as the two form a Fourier transform pair. In simple approaches considering only the inertial and large-scale dynamics, the auto-correlation function is well approximated by $R(t)=\left\langle\left(u^{\prime}\right)^{2}\right\rangle \exp \left(-t / T_{L}\right)$ (Hinze 1975; Mordant et al. 2001; Zhang et al. 2019), valid for $\omega \ll \pi / \tau_{\eta}$ (or $\tau_{\eta} \ll$ $t$ ) and corresponding to $E=\left\langle\left(u^{\prime}\right)^{2}\right\rangle T_{L} /\left(1+\left(\omega T_{L}\right)^{2}\right)$. For small time separations, the autocorrelation deviates from the exponential, tending to a horizontal asymptote at $t=0$ with a curvature proportional to the acceleration variance (Mordant et al. 2004). Sawford (1991) proposed an alternative formulation valid for all $t$, using a second time scale $T_{2}$ 


\section{T. Berk and F. Coletti}

related to the finite acceleration variance

$$
R(t)=\left\langle\left(u^{\prime}\right)^{2}\right\rangle \frac{T_{L} \exp \left(-t / T_{L}\right)-T_{2} \exp \left(-t / T_{2}\right)}{T_{L}-T_{2}},
$$

Fourier transformation yields

$$
E(\omega)=\frac{\left\langle\left(u^{\prime}\right)^{2}\right\rangle}{T_{L}-T_{2}}\left[\frac{T_{L}^{2}}{1+\left(\omega T_{L}\right)^{2}}-\frac{T_{2}^{2}}{1+\left(\omega T_{2}\right)^{2}}\right] .
$$

Note that in this two-time-scale model $T_{L}$ no longer equals the correlation time. We use this expression to model the Lagrangian spectrum of the sampled-fluid velocity, by substituting the respective time scales $T_{L, f p}$ and $T_{2, f p}$. Using the response function (1.10) in combination with (1.8) and (1.9), we have

$$
\begin{gathered}
\left\langle\left(\boldsymbol{u}_{\boldsymbol{p}}{ }^{\prime}\right)^{2}\right\rangle=\left\langle\left(\boldsymbol{u}_{f p}{ }^{\prime}\right)^{2}\right\rangle\left[1-\frac{S t^{2}}{\left(T_{L, f p} / \tau_{\eta}+S t\right)\left(T_{2, f p} / \tau_{\eta}+S t\right)}\right], \\
\left\langle\left(\boldsymbol{a}_{p}{ }^{\prime}\right)^{2}\right\rangle=\tau_{\eta}^{-2}\left\langle\left(\boldsymbol{u}_{f p}{ }^{\prime}\right)^{2}\right\rangle \frac{1}{\left(T_{L, f p} / \tau_{\eta}+S t\right)\left(T_{2, f p} / \tau_{\eta}+S t\right)} .
\end{gathered}
$$

\subsection{Time scales of the sampled-fluid velocity}

Because evaluating equations (4.3) and (4.4) requires estimates for $T_{L, f p}$ and $T_{2, f p}$, we consider the issue of how those compare to $T_{L}$ and $T_{2}$. For tracers, the Lagrangian integral time scale is related to the Eulerian integral time scale $\left(T_{E}\right)$ as (Yeung 2001)

$$
T_{L} / T_{E} \approx 5 / C_{0}
$$

where $C_{0}$ is the pre-factor in the expression of the Lagrangian velocity structure function. This depends on the turbulence Reynolds number, and based on a review of the literature Lien \& D'Asaro (2002) suggested $C_{0}=C_{0}^{\infty}\left(1-\left(0.1 R e_{\lambda}\right)^{1 / 2}\right)$ with $C_{0}^{\infty}=6 \pm 0.5$ (Ouellette et al. 2006). In addition, the numerical prefactor in (4.5) may depend on Reynolds number and large-scale properties of the turbulence (see, for example Sreenivasan 1998; Vassilicos 2015). Considering inertial particles, the Lagrangian integral time scale of the sampled fluid $T_{L, f p}$ is influenced by both inertia and gravity. For the range of $S t$ in the present study, however, the effect of $S t$ as derived empirically by Wang \& Stock (1993) is negligible. (Alternatively, Jung, Yeo \& Lee (2008) proposed an empirical expression of the $S t$ effect derived in zero gravity.) Gravity decreases $T_{L, f p}$ due to the crossing-trajectories effect, for which we adopt the expression proposed by Csanady (1963) (see also Pozorski \& Minier 1998)

$$
T_{L, f p}=T_{L} \frac{1}{\left[1+\alpha\left(T_{L} / T_{E}\right)^{2} S v^{2}\left(u_{\eta} / u_{r m s}\right)^{2}\right]^{1 / 2}},
$$

where $\alpha=1$ and 4 for the time scales associated with the vertical and horizontal components, respectively. Alternatively, using $\left\langle\left(u_{f}^{\prime}\right)^{2}\right\rangle / u_{\eta}^{2}=R e_{\lambda} / \sqrt{15}$ (Hinze 1975) and $T_{L} / \tau_{\eta}=2\left(\operatorname{Re}_{\lambda}+32\right) /\left(\sqrt{15} C_{0}\right)$ (Zaichik, Simonin \& Alipchenkov 2003), this can be expressed as

$$
\frac{T_{L, f p}}{\tau_{\eta}}=\frac{2\left(R e_{\lambda}+32\right)}{\sqrt{15} C_{0}} \frac{1}{\left[1+\alpha\left(5 / C_{0}\right)^{2} S v^{2}\left(\sqrt{15} / R e_{\lambda}\right)^{2}\right]^{1 / 2}},
$$

which is a function of $\operatorname{Re}_{\lambda}$ and $S v$ only. 

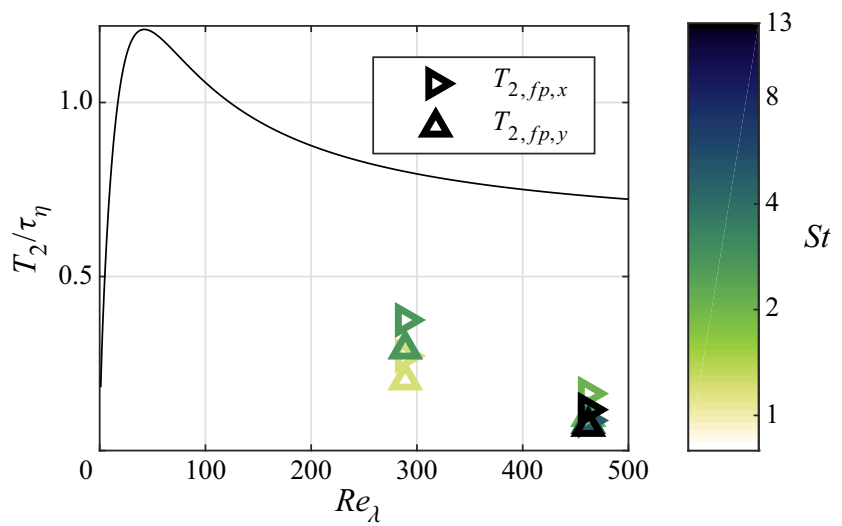

Figure 12. Empirical values of $T_{2, f p}$, determined using (4.9). The black line indicates the prediction for tracers in (4.8).

For tracers, the time scale $T_{2}$ can be written as (Sawford 1991)

$$
T_{2} / \tau_{\eta}=C_{0} /\left(2 a_{0}\right)
$$

A relation for $T_{2, f p}$ is obtained from the variance of $\mathrm{d} u_{f p} / \mathrm{d} t$

$$
\left\langle\left(\frac{\mathrm{d} \boldsymbol{u}_{f p}}{\mathrm{~d} t}\right)^{2}\right\rangle=\frac{2}{\pi} \int_{0}^{\infty} \omega^{2} \boldsymbol{E}_{f p}(\omega) \mathrm{d} \omega=\frac{\left\langle\left(\boldsymbol{u}_{f p}{ }^{\prime}\right)^{2}\right\rangle}{T_{L, f p} T_{2, f p}} .
$$

Thus, we can empirically evaluate $T_{2, f p}$ using the measured values for $\left\langle\left(\mathrm{d} \boldsymbol{u}_{f p}^{\prime} / \mathrm{d} t\right)^{2}\right\rangle$ and $\left\langle\left(\boldsymbol{u}_{f p}{ }^{\prime}\right)^{2}\right\rangle$. The values are presented in figure 12, along with the prediction for tracers in (4.8); $T_{2, f p}$ is measurably smaller than $T_{2}$, although no clear trend with $S t$ is discerned.

Note that (4.9) relates the two time scales by a Taylor time scale of the sampled flow, which can be defined as $\tau_{f p, \lambda}^{2} \equiv\left\langle\left(\boldsymbol{u}_{f p}{ }^{\prime}\right)^{2}\right\rangle /\left\langle\left(\mathrm{d} \boldsymbol{u}_{f p}^{\prime} / \mathrm{d} t\right)^{2}\right\rangle$ (Tennekes \& Lumley 1972; Pope 2000), such that $\tau_{f p, \lambda}^{2}=T_{L, f p} T_{2, f p}$.

With the above estimates of $T_{L, f p}$ and $T_{2, f p}$, we use (4.1) to model the autocorrelations of the sampled-fluid velocity, $R_{f p}$. We consider both horizontal and vertical components, and present the results as correlation coefficients, $\rho_{f p}$, normalizing by the variance of the sampled-fluid velocity for the respective components. The modelled curves are plotted in figure 13, along with the corresponding measurements and the fluid velocity autocorrelation (which is also modelled via (4.1), using $T_{L}$ and $T_{2}$ ). For clarity we separate both $R e_{\lambda}$ cases, as these have different fluid time scales. The plots only extend to values of $t$ for which the measured autocorrelations are based on at least 100 trajectories. This allows for limited time lags, still sufficient to highlight the trends. The sampled-fluid velocity decorrelates faster with $S v$ due to the crossing-trajectory effect. Despite quantitative differences with the measurements, (4.1) captures well this trend for short times. In figure 14 we compare the measured $\rho_{f p}$ against the particle velocity autocorrelation coefficients, $\rho_{p}$; the fluid velocity autocorrelation is also included for reference. The apparent trend is that $\rho_{p}$ decays more slowly for heavier and heavier particles, which is a consequence of inertia; while $\rho_{f p}$ decays more rapidly, which is a consequence of gravity. 


\section{T. Berk and F. Coletti}

(a)

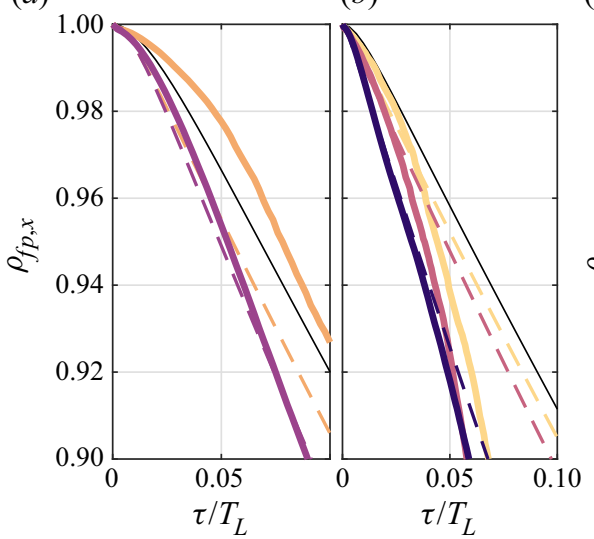

(c)

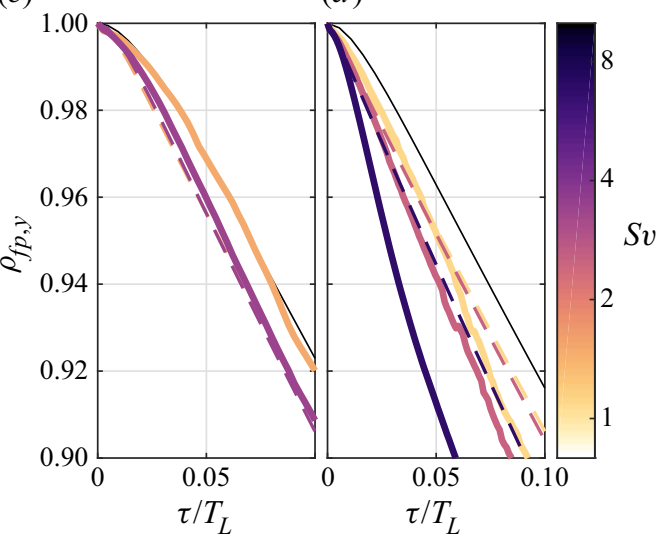

Figure 13. Autocorrelation of the sampled-fluid velocity. Horizontal components for the $R e_{\lambda}=289(a)$ and 462 (b) cases; vertical components for the $R e_{\lambda}=289$ (c) and $462(d)$ cases. Thick solid lines indicate the measured autocorrelation. Thin black lines indicate the prediction for tracers in (4.1). Dashed lines indicate the prediction from (4.1) with corrected time scales for inertial particles using (4.7) and (4.9).

(a)

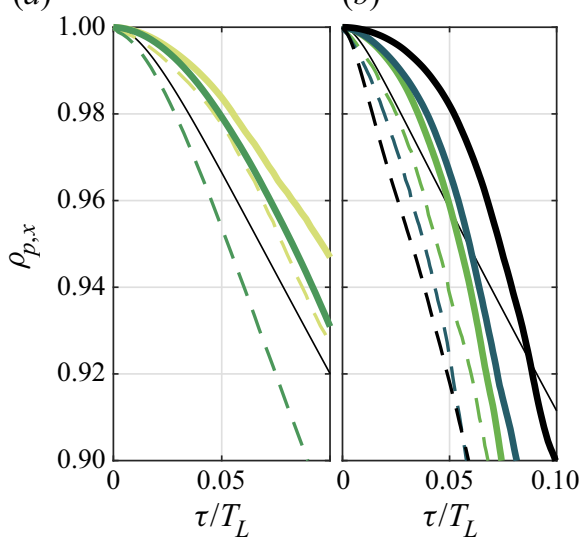

(c)

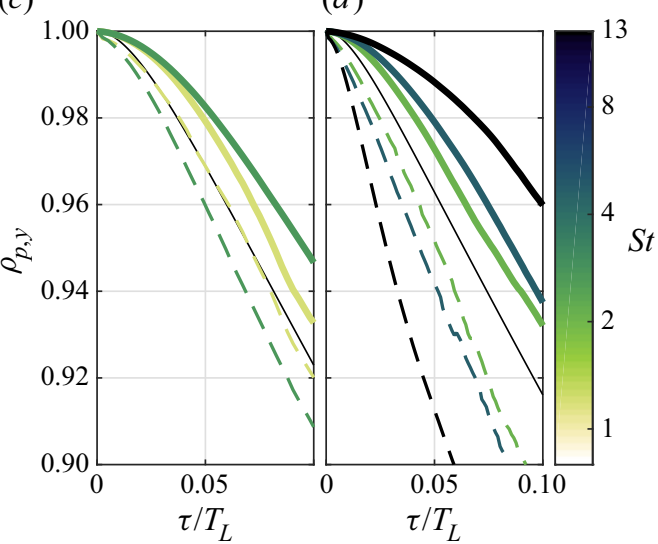

Figure 14. Autocorrelation of the particle velocity. Horizontal components for the $R e_{\lambda}=289$ (a) and 462 (b) cases; vertical components for the $R e_{\lambda}=289$ (c) and $462(d)$ cases. Thick solid lines indicate the autocorrelation. Thin black lines indicate the prediction for tracers in (4.1). Dashed lines indicate the autocorrelation of the particle-sampled-fluid velocity as presented in figure 13 .

\subsection{Model predictions}

We now compare the measured variances of the particle velocity and particle acceleration against the respective predictions from (4.3) and (4.4). In non-dimensional form

$$
\begin{aligned}
& \frac{\left\langle\left(\boldsymbol{u}_{\boldsymbol{p}}\right)^{2}\right\rangle}{\left\langle\left(\boldsymbol{u}_{f p^{\prime}}\right)^{2}\right\rangle}=1-\frac{S t^{2}}{\left(T_{L, f p} / \tau_{\eta}+S t\right)\left(T_{2, f p} / \tau_{\eta}+S t\right)}, \\
& \frac{\left\langle\left(\boldsymbol{a}_{\boldsymbol{p}}\right)^{2}\right\rangle}{\left\langle\left(\boldsymbol{u}_{f p^{\prime}}\right)^{2}\right\rangle \tau_{\eta}^{-2}}=\frac{1}{\left(T_{L, f p} / \tau_{\eta}+S t\right)\left(T_{2, f p} / \tau_{\eta}+S t\right)} .
\end{aligned}
$$



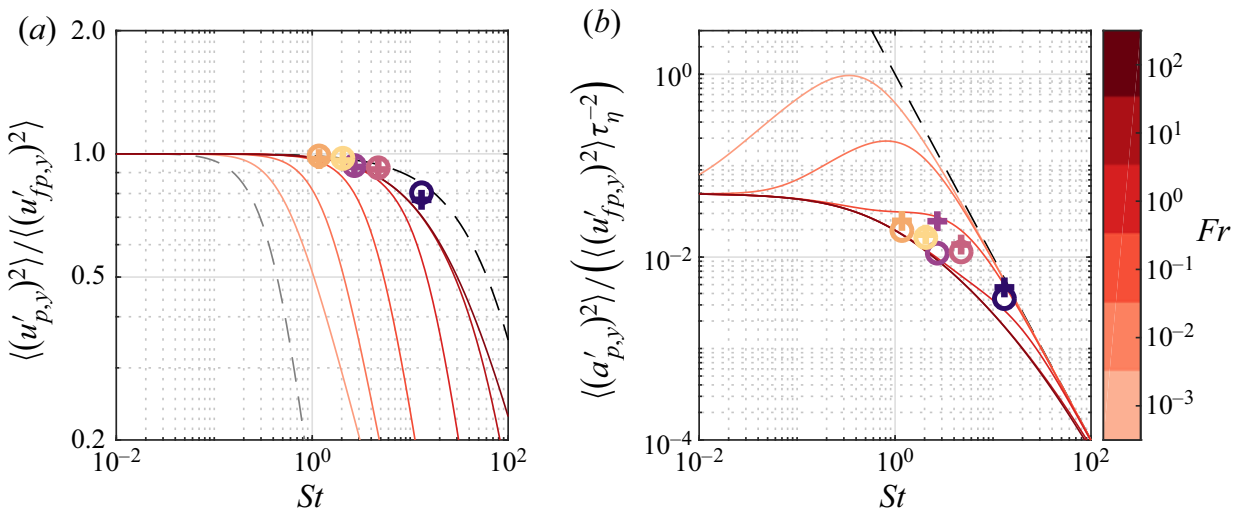

Figure 15. Modelled variances of the vertical component of the particle velocity $(a)$ and acceleration $(b)$. Red lines indicate the predictions from (4.10) and (4.11), coloured by Fr. Circles indicated measured data, coloured by $S v$ (see, for example figure 13 for colour coding). The ' + ' signs indicate case-specific predictions as discussed in the text for matching colours. Grey and black dashed lines in $(a)$ indicate results from the model by Ayala et al. (2008) for $F r=10^{-3}$ and $10^{2}$ respectively. The dashed line in $(b)$ indicates the asymptote $S t^{-2}$.

These are shown in figures $15(a)$ and $15(b)$, respectively, as a function of St. The expressions are essentially model forms of the particle response function, as they describe the variance of the particle velocity and acceleration with respect to the sampled-fluid flow. Here, and in the following, we again consider the vertical components only, the horizontal components leading to analogous conclusions. These 'generalized predictions' are compared with the measurements and with 'case-specific predictions'. The latter differ from the generalized predictions due to the difference in $T_{2, f p}$ : for the generalized model we approximate $T_{2, f p} \approx T_{2}$ (which is calculated for any St according to (4.8)), whereas for the case-specific predictions we use the empirically determined values for $T_{2, f p}$ (figure 12). As can be seen, this simplification has a moderate impact and does not alter the trends. In the small $S t$ limit, the modelled particle velocity variance equals the variance of the sampled-fluid velocity, regardless of $F r$. For finite $S t$, the particle velocity variance $\left\langle\left(u_{p}^{\prime}\right)^{2}\right\rangle /\left\langle\left(u_{f p}^{\prime}\right)^{2}\right\rangle$ decreases with both inertia and gravity (figure 15a). The dependence with $S t$ reflects inertial filtering, while the effect of $F r$ reflects the decrease of $T_{L, f p}$ with gravitational drift, which in turn damps $\left\langle\left(u_{p}^{\prime}\right)^{2}\right\rangle(4.10)$. The effect of gravity becomes negligible for $\mathrm{Fr} \gtrsim 10$. In the limits of either strong gravity $(F r \ll 1)$ or large inertia $(S t \gg 1),\left\langle\left(u_{p}^{\prime}\right)^{2}\right\rangle$ vanishes compared to $\left\langle\left(u_{f p}^{\prime}\right)^{2}\right\rangle$. Overall, the generalized model represents well the present data. Modelled results are qualitatively similar to results of the model presented in Ayala, Rosa \& Wang (2008), the main quantitative difference resulting from the omission of a second time scale $\left(T_{2}\right)$ in that model.

The normalized particle acceleration variance $\left\langle\left(a_{p}^{\prime}\right)^{2}\right\rangle /\left(\left\langle\left(u_{f p}^{\prime}\right)^{2}\right\rangle \tau_{\eta}^{-2}\right)$ (figure 15b) tends to $a_{0} / u_{0}$ for small $S t$, where $u_{0} \equiv\left\langle a_{f}^{2}\right\rangle / u_{\eta}^{2}$ is a function of $R e_{\lambda}$. For large $S t$, it asymptotes to $S t^{-2}$, or $\left\langle\left(a_{p}^{\prime}\right)^{2}\right\rangle=\left\langle\left(u_{f p}^{\prime}\right)^{2}\right\rangle \tau_{p}^{-2}$. For $F r \ll 1$ the particle acceleration variance displays a non-monotonic behaviour with $S t$, due to the competing effects of inertia and gravity: an increase of inertia for a fixed $F r$ implies an increase of $S v$ and therefore of crossing-trajectory drift, augmenting $\mathrm{d} u_{f p, y} / \mathrm{d} t$ and in turn also the particle acceleration. As $S t$ increases further, inertial filtering eventually dominates and the particle acceleration is dampened. 


\section{T. Berk and F. Coletti}
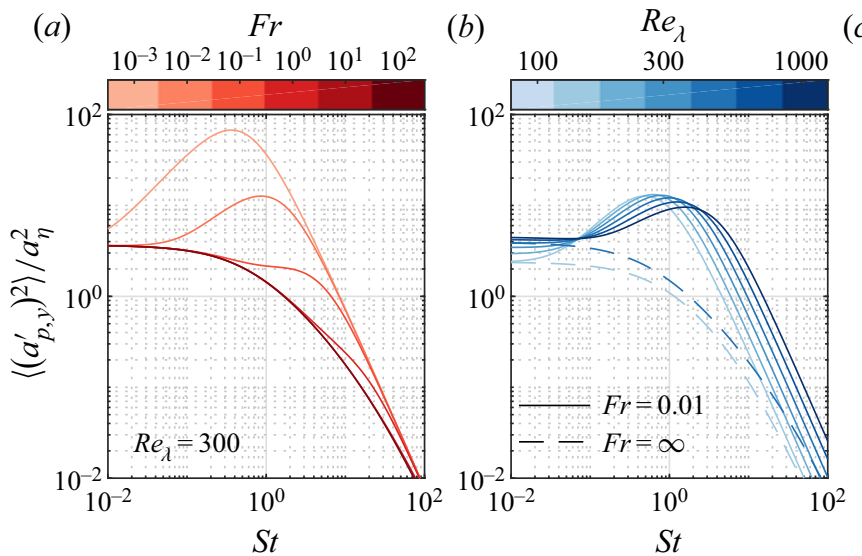

(c)

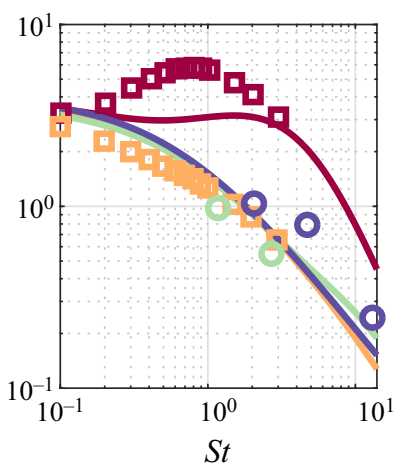

Figure 16. Vertical component of the particle acceleration variance, modelled using (4.12). Variation in $\mathrm{Fr}$ for $R e_{\lambda}=300(a)$. Variation in $R e_{\lambda}$ for $F r=0.01$ (solid lines) and $F r=\infty$ (dashed lines) (b). Comparison of cases from Ireland et al. (2016b) (squares) and the present study (circles) with the prediction in (4.12) (lines) (c). Matching colours have matched $\mathrm{Fr}$ and $\operatorname{Re}_{\lambda}$.

As the sampled-fluid properties are not known a priori, (4.10) and (4.11) have limited predictive power. However, as shown in figure 5, the sampled-fluid velocity variance is marginally smaller than the fluid velocity variance, and one can approximate $\left\langle\left(u_{f}^{\prime}\right)^{2}\right\rangle \approx$ $\left\langle\left(u_{f p}^{\prime}\right)^{2}\right\rangle$. Taking $\left\langle\left(u_{f}^{\prime}\right)^{2}\right\rangle / u_{\eta}^{2}=R e_{\lambda} / \sqrt{15}$ (Hinze 1975) and substituting in (4.11) leads to

$$
\frac{\left\langle\left(a_{p}{ }^{\prime}\right)^{2}\right\rangle}{a_{\eta}^{2}}=\frac{\operatorname{Re}_{\lambda}}{\sqrt{15}} \frac{1}{\left(T_{L, f p} / \tau_{\eta}+S t\right)\left(T_{2} / \tau_{\eta}+S t\right)},
$$

where we assumed $T_{2, f p} \approx T_{2}$ and $T_{L, f p}$ is obtained from (4.6). Because of the assumptions made, (4.12) is expected to underpredict somewhat the observations. The dependence on $S t, F r$ and $R e_{\lambda}$ is illustrated in figure 16. For large $S t$, the curves asymptote to $\operatorname{Re}_{\lambda} S t^{-2} / \sqrt{15}$. At intermediate $S t$ and $F r \ll 1$, we retrieve the non-monotonic behaviour discussed above. This non-monotonicity was also reported by Ireland et al. (2016b) for $F r=0.052$ as well as by Parishani et al. (2015) for $F r \approx 0.14$. In general (4.12) agrees remarkably well with the trends reported by their simulations as well as with our experimental data. The present model demonstrates how the value of $S t$ that maximizes the particle acceleration increases with $F r$, while the maximum shrinks and eventually disappears for $F r \gtrsim 0.1$. This is the reason why the non-monotonic behaviour found by Ireland et al. (2016b) at $F r=0.052$ is not seen in our study at $F r=O(1)$, nor in previous studies at $F r \approx 0.3$ (Ayyalasomayajula et al. 2006) and $F r \approx 30$ (Volk et al. 2008). For increasing $R e_{\lambda}$, the maximum of $\left\langle\left(a_{p}^{\prime}\right)^{2}\right\rangle / a_{\eta}^{2}$ is found at larger St. This is consistent with the view that, with increasing $R e_{\lambda}$, a wider range of scales may influence the particle motion, and therefore also particles with $S t>O(1)$ may respond strongly to the turbulence (Yoshimoto \& Goto 2007).

We now use (4.11) to derive an expression for the slip velocity variance. Squaring and averaging the fluctuating part of the particle equation of motion, $\boldsymbol{a}_{\boldsymbol{p}}{ }^{\prime}=-\boldsymbol{u}_{\boldsymbol{s}}{ }^{\prime} / \tau_{p}$, we have $\left\langle\left(\boldsymbol{u}_{\boldsymbol{s}}{ }^{\prime}\right)^{2}\right\rangle=\tau_{p}^{2}\left\langle\left(\boldsymbol{a}_{\boldsymbol{p}}{ }^{\prime}\right)^{2}\right\rangle$. Substituting in (4.11) gives

$$
\frac{\left\langle\left(\boldsymbol{u}_{\boldsymbol{s}}\right)^{2}\right\rangle}{\left\langle\left(\boldsymbol{u}_{f p}{ }^{\prime}\right)^{2}\right\rangle}=\frac{S t^{2}}{\left(T_{L, f p} / \tau_{\eta}+S t\right)\left(T_{2} / \tau_{\eta}+S t\right)},
$$



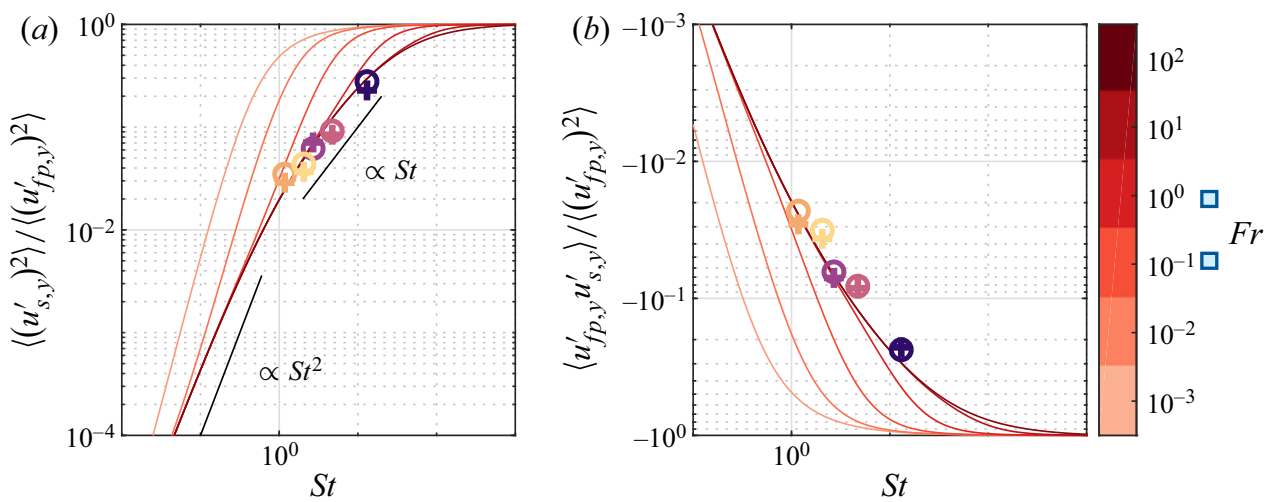

Figure 17. Vertical components of the modelled variance of the slip velocity $(a)$ and covariance of the slip velocity and the sampled-fluid velocity $(b)$. Red lines indicate the predictions from (4.13) and $\left\langle\boldsymbol{u}_{f p}{ }^{\prime} \boldsymbol{u}_{\boldsymbol{s}}{ }^{\prime}\right\rangle=$ $-\left\langle\left(\boldsymbol{u}_{s}{ }^{\prime}\right)^{2}\right\rangle$, coloured by $F r$. Circles indicated measured data, coloured by $S v$ (see, for example figure 13 for colour coding). The ' + ' signs indicate case-specific predictions as discussed in the text for matching colours.

where again we assumed $T_{2, f p} \approx T_{2}$ and $T_{L, f p}$ is obtained from (4.6). Figure 17(a) verifies this prediction, comparing it with the case-specific predictions (using empirical estimates for $T_{2}$ ) and the measurements. The modelled slip velocity variance is in the ranges $\left\langle\left(u_{s, y}^{\prime}\right)^{2}\right\rangle \approx 0$ in the small St limit, when particles faithfully follow the flow; to $\left\langle\left(u_{s, y}^{\prime}\right)^{2}\right\rangle \approx\left(u_{f p, y}^{\prime}\right)^{2}$ at large $S t$, when particles are ballistic and the slip velocity fluctuations effectively equal the velocity fluctuations of the sampled fluid. In between, the variance of the slip velocity increases with both inertia and gravity as both effects reduce the ability of the particles to follow the fluid fluctuations. The agreement with the measurements is remarkable. Also, the generalized model is consistent with the scaling derived by Balachandar (2009) for the different regimes: for the slip velocity variance, his arguments imply $\left\langle\left(u_{s, y}^{\prime}\right)^{2}\right\rangle \propto S t^{2}$ for $\tau_{p}<\tau_{\eta},\left\langle\left(u_{s, y}^{\prime}\right)^{2}\right\rangle \propto S t$ for $\tau_{\eta}<\tau_{p}<T_{L}$, and $\left\langle\left(u_{s, y}^{\prime}\right)^{2}\right\rangle \approx$ constant for $\tau_{p}>T_{L}$. Figure 17(b) shows that the covariance of the sampled-fluid velocity and slip velocity is approximately $\left\langle\boldsymbol{u}_{f p}{ }^{\prime} \boldsymbol{u}_{\boldsymbol{s}}{ }^{\prime}\right\rangle \approx-\left\langle\left(\boldsymbol{u}_{\boldsymbol{s}}{ }^{\prime}\right)^{2}\right\rangle$. This result is supported by the measurements (see figure 4$)$, and can be derived from the assumptions of the model: comparing (4.13) and (4.10) implies $\left\langle\left(\boldsymbol{u}_{\boldsymbol{p}}{ }^{\prime}\right)^{2}\right\rangle=\left\langle\left(\boldsymbol{u}_{f \boldsymbol{p}}{ }^{\prime}\right)^{2}\right\rangle-\left\langle\left(\boldsymbol{u}_{\boldsymbol{s}}{ }^{\prime}\right)^{2}\right\rangle$, and comparing the latter equality with the variance of the particle velocity (expressed as $\left.\boldsymbol{u}_{\boldsymbol{p}}=\boldsymbol{u}_{f \boldsymbol{p}}+\boldsymbol{u}_{\boldsymbol{s}}\right)$ implies $\left\langle\boldsymbol{u}_{f p}{ }^{\prime} \boldsymbol{u}_{\boldsymbol{s}}{ }^{\prime}\right\rangle=-\left\langle\left(\boldsymbol{u}_{\boldsymbol{s}}{ }^{\prime}\right)^{2}\right\rangle$. Accordingly, the covariance ranges from approximately zero at small $S t$, where there is no slip velocity, to $\left\langle\boldsymbol{u}_{f p}{ }^{\prime} \boldsymbol{u}_{\boldsymbol{s}}{ }^{\prime}\right\rangle=\left\langle\left(\boldsymbol{u}_{f p}{ }^{\prime}\right)^{2}\right\rangle$ at large $S t$, where the slip velocity equals the sampled-fluid velocity.

From the relationship $\left\langle\boldsymbol{u}_{f p}{ }^{\prime} \boldsymbol{u}_{\boldsymbol{s}}{ }^{\prime}\right\rangle \approx-\left\langle\left(\boldsymbol{u}_{\boldsymbol{s}}{ }^{\prime}\right)^{2}\right\rangle$ (and substituting $\boldsymbol{u}_{\boldsymbol{s}}{ }^{\prime}=\tau_{p} \boldsymbol{a}_{p}{ }^{\prime}$ and/or $\left.\boldsymbol{u}_{\boldsymbol{s}}{ }^{\prime}=\boldsymbol{u}_{p}{ }^{\prime}-\boldsymbol{u}_{f p}{ }^{\prime}\right)$ we can derive other covariances between the particle velocity, the sampled-fluid velocity, and the particle acceleration; those are especially useful to formulate stochastic models (Zamansky, Vinkovic \& Gorokhovski 2013; Pope 2014). Normalizing by the respective r.m.s. values, we have expressions for the following correlation coefficients:

$$
\rho\left(\boldsymbol{u}_{p} \boldsymbol{u}_{f p}\right)=\sqrt{1-\frac{S t^{2}}{\left(T_{L, f p} / \tau_{\eta}+S t\right)\left(T_{2} / \tau_{\eta}+S t\right)}},
$$




\section{T. Berk and F. Coletti}
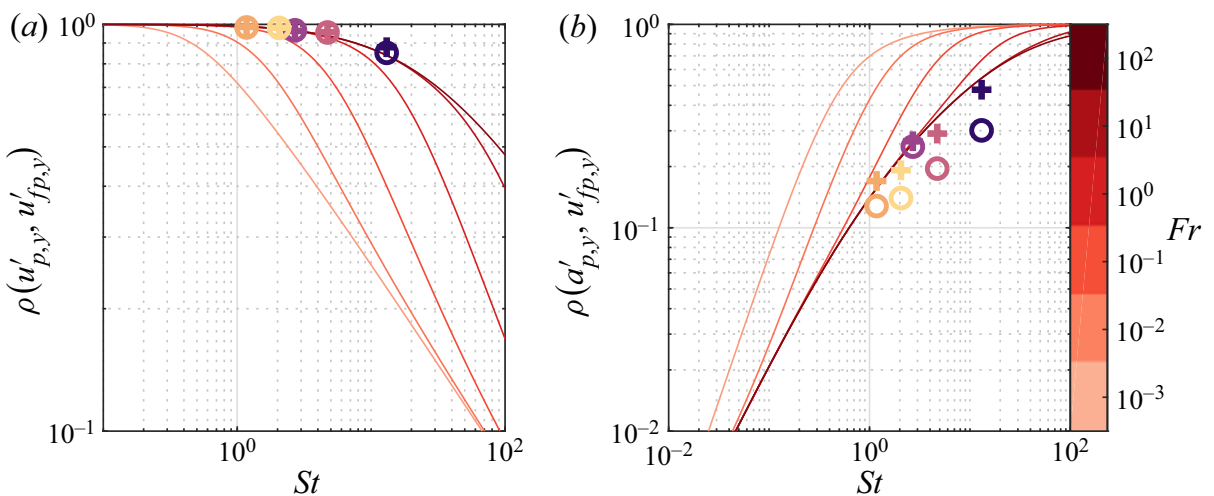

Figure 18. Modelled correlation coefficients of the vertical components of the particle velocity and sampled-fluid velocity $(a)$ and of the particle acceleration and sampled-fluid velocity $(b)$. Red lines indicate the predictions from (4.14) and (4.15), coloured by Fr. Circles indicated measured data, coloured by $S v$ (see, for example figure 13 for colour coding). The '+' signs indicate case-specific predictions as discussed in the text for matching colours.

$$
\begin{gathered}
\rho\left(\boldsymbol{a}_{\boldsymbol{p}} \boldsymbol{u}_{\boldsymbol{p} \boldsymbol{p}}\right)=\sqrt{\frac{S t^{2}}{\left(T_{L, f p} / \tau_{\eta}+S t\right)\left(T_{2} / \tau_{\eta}+S t\right)}}, \\
\rho\left(\boldsymbol{a}_{\boldsymbol{p}} \boldsymbol{u}_{\boldsymbol{p}}\right)=0 .
\end{gathered}
$$

The model predictions in (4.14) and (4.15) are presented in figure 18 for the vertical component. The correlation between the particle velocity and sampled-fluid velocity, $\rho\left(\boldsymbol{u}_{\boldsymbol{p}} \boldsymbol{u}_{f p}\right)$, approximates unity in the small $\boldsymbol{S t}$ limit as expected, and the model predicts the decorrelation due to inertia and gravity (figure 18a). The agreement with the measurements is satisfactory, although a comparison with data at lower $\mathrm{Fr}$ is needed to corroborate the prediction. In contrast, the particle acceleration does not correlate with the fluid velocity at small $S t$ (figure $18 b)$, with $\rho\left(\boldsymbol{a}_{p} \boldsymbol{u}_{f p}\right)$ increasing with gravity and inertia. Indeed, in the ballistic limit, the slip velocity fluctuations are equal and opposite to the fluid velocity fluctuations, that is $\boldsymbol{a}_{p}{ }^{\prime}=-\boldsymbol{u}_{\boldsymbol{s}}{ }^{\prime} / \tau_{p}=\boldsymbol{u}_{f p}{ }^{\prime} / \tau_{p}$. The significant mismatch with the data (approximately $40 \%$ for most of the cases) may partly be due to inherent uncertainty on the particle acceleration, but is also likely related to the simplistic assumption that drag and gravity are the only forces at play. Finally, the prediction that the particle acceleration is uncorrelated from the particle velocity (4.16) is confirmed by our measurements, from which we find $\rho\left(\boldsymbol{a}_{\boldsymbol{p}} \boldsymbol{u}_{\boldsymbol{p}}\right)<0.05$ for all cases.

\section{Discussion and conclusions}

We have experimentally investigated the transport of sub-Kolmogorov heavy particles in homogeneous turbulence. All relevant spatio temporal scales are resolved for the first time in a similar configuration, albeit through planar measurements. We consider ranges of $S t$ and $S v$ for which a rich particle-turbulence interaction is expected, including settling enhancement, inertial filtering and preferential sampling. The focus is on the respective roles of inertia and gravity, which have different and often competing influence on the particle motion. A unique feature of the present measurements is the access to the local properties of the turbulence experienced by the particles along their trajectories.

The importance of the sampled-fluid properties is already clear from the mean vertical velocities. It is proposed that, in the present ranges of $S t$ and $S v$, the particle settling 


\section{Dynamics of small heavy particles in homogeneous turbulence}

velocity can be rationalized by assuming $\left\langle u_{f p, y}\right\rangle / u_{\eta}=C$. Because, to first order, the settling enhancement $\Delta u_{y}=\left\langle u_{f p, y}\right\rangle$, it is also proportional to the velocity scale of the turbulence, consistent with previously reported trends. The limited range of $R e_{\lambda}$, however, cannot clarify whether this scale shall be $u_{\eta}, u_{r m s}$, or a multi-scale quantity between those. The mean of the instantaneous slip velocity is well represented by Stokes drag with the Schiller \& Neumann correction, at least for moderate St. Upward- and downward-moving particles sample fluid regions with large velocity fluctuations in those same directions, with similar magnitude in both cases. Therefore, the settling velocity is augmented due to the downward-moving particles being more numerous, not because the sampled downward fluid fluctuations are stronger than the upward ones.

The fluid fluctuations also play a dominant role in determining the particle velocity fluctuations. The variance of the particle velocity $\left\langle\left(\boldsymbol{u}_{p}{ }^{\prime}\right)^{2}\right\rangle$ is comparable to but somewhat smaller than the sampled-fluid velocity variance $\left\langle\left(\boldsymbol{u}_{f p}{ }^{\prime}\right)^{2}\right\rangle$, due to inertial filtering; and the latter is slightly smaller than the fluid velocity variance $\left\langle\left(\boldsymbol{u}_{f}^{\prime}\right)^{2}\right\rangle$, due to gravitational drift. While gravity and inertia have concurrent effects on the particle fluctuating energy, their influences on the particle accelerations are opposite to each other: the crossing-trajectories effect augments the temporal derivative of the sampled-fluid velocity, $\left\langle\left(\mathrm{d} \boldsymbol{u}_{f p}{ }^{\prime} / \mathrm{d} t\right)^{2}\right\rangle$, which act to enhance the particle acceleration variance; but this is offset (at least in the present range of parameters) by inertial filtering. The net result is that heavier particles display smaller r.m.s. accelerations and less intermittent acceleration p.d.f.s. The preferential sampling of high-strain/low-vorticity regions is measurable, but its global impact on the particle motion is weak.

The competing influences of inertia and gravity are on display also in the two-particle statistics. The uncorrelated component of the relative motion augments the particle velocity structure functions at small separations; while the reduced fluctuating energy of the particles (compared to tracers) has an opposite effect at inertial-range and large-scale separations. The large relative velocities of nearby particles, which increase with particle inertia, cause heavier particles to separate faster; still, the mean square separation is generally below the expectation for tracers. This is attributed to gravity causing the particles to experience fluid velocities that decorrelate faster in time, with respect to zero-gravity conditions. The inertial particles appear to transition out of the ballistic regime at earlier times compared to tracers, similarly to what recently shown for bubbles rising in homogeneous turbulence.

The planar nature of the measurements limits the full characterization of both the particle motion and the turbulent fluid flow. This is expected to affect especially the two-particle statistics in the form of a biased sampling of the trajectories, although this may not obscure the apparent trends. The three-dimensional tracking of the particles would overcome this limitation. Nevertheless, volumetric techniques are presently not capable of simultaneously capturing both phases at the required resolution: despite fast-paced advances in this area, the spatial resolution in volumetric PIV/PTV is still generally below what can be achieved by planar measurements (Discetti \& Coletti 2018); nor these methods have systematically been adapted to multi-phase flows yet. The present study indeed highlights the central role of the sampled-fluid properties, and extending such an analysis to three-dimensional experiments remains a challenge.

Based on these experimental observations, we have derived an analytical model of particle velocity and acceleration inspired by the seminal work of Csanady (1963). This is based on applying a response function to the spectrum of fluid velocities experienced by the particles. To this end, we use the expression proposed by Sawford (1991) for the fluid velocity autocorrelation, in which we substitute estimates for the time scales $T_{L}$ and 


\title{
T. Berk and F. Coletti
}

$T_{2}$ of the sampled fluid. We lack an analytical expression for the latter time scale, but we show that using the unconditional-fluid formulation (with classic estimates of the $C_{0}$ constant) has a small quantitative influence. In its basic form ((4.10) and (4.11)) the model provides the particle velocity and acceleration variances as a function of $\left\langle\left(\boldsymbol{u}_{f p}{ }^{\prime}\right)^{2}\right\rangle$. The model agrees generally well with the experimental observations, captures the respective effects of inertia and gravity over a wide range of the controlling parameters, and predicts correlations between particle and sampled-fluid velocities and accelerations. In particular, consistent with the arguments by Balachandar (2009), it predicts the variance of the slip velocity $\left\langle\left(\boldsymbol{u}_{\boldsymbol{s}}{ }^{\prime}\right)^{2}\right\rangle$ to scale as $S t^{2}$ for $\tau_{p}<\tau_{\eta}$, as $S t$ for $\tau_{\eta}<\tau_{p}<T_{L}$, and to plateau for $\tau_{p}>T_{L}$.

Because we show that $\left\langle\left(\boldsymbol{u}_{f \boldsymbol{p}}{ }^{\prime}\right)^{2}\right\rangle$ is only slightly smaller than $\left\langle\left(\boldsymbol{u}_{\boldsymbol{f}}{ }^{\prime}\right)^{2}\right\rangle$ (at least in the present range of parameters), the model can be written in a weaker form with more predictive power by substituting $\left\langle\left(u_{f p}{ }^{\prime}\right)^{2}\right\rangle=u_{\eta}^{2} R e_{\lambda} / \sqrt{15}$ (Hinze 1975)

$$
\begin{gathered}
\frac{\left\langle\left(\boldsymbol{u}_{\boldsymbol{p}}{ }^{\prime}\right)^{2}\right\rangle}{u_{\eta}^{2}}=\frac{\operatorname{Re} \lambda}{\sqrt{15}}\left[1-\frac{S t^{2}}{\left(T_{L, f p} / \tau_{\eta}+S t\right)\left(T_{2} / \tau_{\eta}+S t\right)}\right], \\
\frac{\left\langle\left(\boldsymbol{a}_{\boldsymbol{p}}{ }^{\prime}\right)^{2}\right\rangle}{a_{\eta}^{2}}=\frac{\operatorname{Re}_{\lambda}}{\sqrt{15}} \frac{1}{\left(T_{L, f p} / \tau_{\eta}+S t\right)\left(T_{2} / \tau_{\eta}+S t\right)},
\end{gathered}
$$

in which $T_{2} / \tau_{\eta}$ is a function of $R e_{\lambda}$ only and $T_{L, f p} / \tau_{\eta}$ is a function of $R e_{\lambda}$ and $S v$ only, given by (4.8) and (4.7), respectively. When compared with the present experiments and recent simulations, in particular for the particle acceleration, this version of the model also agrees well with the observations and represents the complex dependency with inertia and gravity. In particular, it predicts the increase in r.m.s. particle acceleration with gravitational drift, and its non-monotonic dependence with $S t$ when $F r \ll 1$ as recently reported by Ireland et al. $(2016 b)$.

Taken together, the laboratory observations and the derived model indicate how, unless the turbulence acceleration is overwhelming $(F r \gg 1)$, both inertia and gravity are key ingredients to understand the transport of heavy particles in homogeneous turbulence. This calls into question the practice of setting gravity to zero to isolate inertial effects. Such a consideration appears to be broadly applicable: recent studies on bubbles in homogeneous turbulence (Mathai et al. 2016) and heavy particles in turbulent boundary layers (Baker \& Coletti 2021) reached a similar conclusion.

Funding. The present work was supported in part by the US Army Research Office, Division of Earth Materials and Processes (grant W911NF-17-1-0366), and Division of Fluid Dynamics (grant W911NF-18-1-0354).

Declaration of interests. The authors report no conflict of interest.

\author{
Author ORCIDs. \\ (1) Tim Berk https://orcid.org/0000-0002-7159-2360; \\ () Filippo Coletti https://orcid.org/0000-0001-5344-2476.
}

\section{REFERENCES}

Aliseda, A., Cartellier, A., Hainaux, F. \& Lasheras, J.C. 2002 Effect of preferential concentration on the settling velocity of heavy particles in homogeneous isotropic turbulence. J. Fluid Mech. 468, 77-105. Ayala, O., RosA, B. \& WANG, L.-P. 2008 Effects of turbulence on the geometric collision rate of sedimenting droplets. Part 2. Theory and parameterization. New J. Phys. 10 (7), 075016. 


\section{Dynamics of small heavy particles in homogeneous turbulence}

Ayyalasomayajula, S., Gylfason, A., Collins, L.R., Bodenschatz, E. \& Warhaft, Z. 2006 Lagrangian measurements of inertial particle accelerations in grid generated wind tunnel turbulence. Phys. Rev. Lett. 97 (14), 144507.

Baker, L., Frankel, A., Mani, A. \& Coletti, F. 2017 Coherent clusters of inertial particles in homogeneous turbulence. J. Fluid Mech. 833, 364-398.

BAKer, L.J. \& COLETTI, F. 2021 Particle-fluid-wall interaction of inertial spherical particles in a turbulent boundary layer. J. Fluid Mech. 908, A39.

BALACHANDAR, S. 2009 A scaling analysis for point-particle approaches to turbulent multiphase flows. Intl J. Multiphase Flow 35 (9), 801-810.

Balachandar, S. \& Eaton, J.K. 2010 Turbulent dispersed multiphase flow. Annu. Rev. Fluid Mech. 42 (1), 111-133

BATCHELOR, G.K. 1950 The application of the similarity theory of turbulence to atmospheric diffusion. Q. J. R. Meteorol. Soc. 76 (328), 133-146.

Bec, J., Biferale, L., Boffetta, G., Celani, A., Cencini, M., Lanotte, A., Musacchio, S. \& Toschi, F. 2006 Acceleration statistics of heavy particles in turbulence. J. Fluid Mech. 550, 349-358.

Bec, J., Biferale, L., Lanotte, A.S., Scagliarini, A. \& Toschi, F. 2010 Turbulent pair dispersion of inertial particles. J. Fluid Mech. 645, 497-528.

Bitane, R., Homann, H. \& Bec, J. 2012 Time scales of turbulent relative dispersion. Phys. Rev. E 86 (4), 045302 .

Bourgoin, M. 2015 Turbulent pair dispersion as a ballistic cascade phenomenology. J. Fluid Mech. 772, 678-704.

BRAGG, A.D. \& Collins, L.R. 2014 New insights from comparing statistical theories for inertial particles in turbulence: II. Relative velocities. New J. Phys. 16 (5), 055014.

Bragg, A.D., Ireland, P.J. \& Collins, L.R. 2016 Forward and backward in time dispersion of fluid and inertial particles in isotropic turbulence. Phys. Fluids 28 (1), 013305.

CARdesa, J.I., Mistry, D., GAn, L. \& DAwson, J.R. 2013 Invariants of the reduced velocity gradient tensor in turbulent flows. J. Fluid Mech. 716, 597-615.

Carter, D., Petersen, A., Amili, O. \& Coletti, F. 2016 Generating and controlling homogeneous air turbulence using random jet arrays. Exp. Fluids 57 (12), 189.

Carter, D.W. \& Coletti, F. 2017 Scale-to-scale anisotropy in homogeneous turbulence. J. Fluid Mech. 827, 250-284.

CARter, D.W. \& Coletti, F. 2018 Small-scale structure and energy transfer in homogeneous turbulence. J. Fluid Mech. 854, 505-543.

Chang, K., Malec, B.J. \& Shaw, R.A. 2015 Turbulent pair dispersion in the presence of gravity. New J. Phys. 17 (3), 033010.

Clift, R., Grace, J. \& Weber, M.E. 2005 Bubbles, Drops and Particles. Dover.

CSAnADY, G.T. 1963 Turbulent diffusion of heavy particles in the atmosphere. J. Atmos. Sci. 20 (3), $201-208$.

Deutsch, E. \& Simonin, O. 1991 Large eddy simulation applied to the modelling of particulate transport coefficients in turbulent two-phase flows. In 8th Symposium on Turbulent Shear Flows, vol. 1, pp. 1011-1016. University of Munich.

DhARIWAL, R. \& BRAGG, A.D. 2019 Enhanced and suppressed multiscale dispersion of bidisperse inertial particles due to gravity. Phys. Rev. Fluids 4 (3), 034302.

Discetti, S. \& Coletti, F. 2018 Volumetric velocimetry for fluid flows. Meas. Sci. Technol. 29 (4), 042001.

Dou, Z., Ireland, P.J., Bragg, A.D., Liang, Z., Collins, L.R. \& Meng, H. 2018 Particle-pair relative velocity measurement in high-Reynolds-number homogeneous and isotropic turbulence using 4-frame particle tracking velocimetry. Exp. Fluids 59 (2), 30.

Ebrahimian, M., Sanders, R.S. \& Ghaemi, S. 2019a Dynamics and wall collision of inertial particles in a solid-liquid turbulent channel flow. J. Fluid Mech. 881, 872-905.

Ebrahimian, M., Sanders, R.S. \& Ghaemi, S. $2019 b$ Near-wall motion of inertial particles in a drag-reduced non-newtonian turbulent flow. Exp. Fluids 60 (7), 117.

Elghobashi, S. \& TRUesdell, G.C. 1992 Direct simulation of particle dispersion in a decaying isotropic turbulence. J. Fluid Mech. 242, 655-700.

Falkinhoff, F., Obligado, M., Bourgoin, M. \& Mininni, P.D. 2020 Preferential concentration of free-falling heavy particles in turbulence. Phys. Rev. Lett. 125 (6), 064504.

FeVrier, P., Simonin, O. \& Squires, D.K. 2005 Partitioning of particle velocities in gas-solid turbulent flows into a continuous field and a spatially uncorrelated random distribution: theoretical formalism and numerical study. J. Fluid Mech. 533, 1-46.

Fiscaletti, D., Ganapathisubramani, B. \& Elsinga, G.E. 2015 Amplitude and frequency modulation of the small scales in a jet. J. Fluid Mech. 772, 756-783. 


\section{T. Berk and F. Coletti}

Fong, K.O., Amili, O. \& Coletti, F. 2019 Velocity and spatial distribution of inertial particles in a turbulent channel flow. J. Fluid Mech. 872, 367-406.

Gerashchenko, S., Sharp, N.S., Neuscamman, S. \& Warhaft, Z. 2008 Lagrangian measurements of inertial particle accelerations in a turbulent boundary layer. J. Fluid Mech. 617, 255-281.

Gibert, M., XU, H. \& BodenschatZ, E. 2010 Inertial effects on two-particle relative dispersion in turbulent flows. Europhys. Lett. 90 (6), 64005.

Good, G.H., Gerashchenko, S. \& WARhaft, Z. 2012 Intermittency and inertial particle entrainment at a turbulent interface: the effect of the large-scale eddies. J. Fluid Mech. 694, 371-398.

Good, G.H., Ireland, P.J., Bewley, G.P., Bodenschatz, E., Collins, L.R. \& Warhaft, Z. 2014 Settling regimes of inertial particles in isotropic turbulence. J. Fluid Mech. 759, R3.

Gustavsson, K. \& Mehlig, B. 2016 Statistical models for spatial patterns of heavy particles in turbulence. Adv. Phys. 65 (1), 1-57.

HiLl, R.J. 2002 Scaling of acceleration in locally isotropic turbulence. J. Fluid Mech. 452, 361-370.

HinZE, J.O. 1975 Turbulence. Mcgraw-Hill Publishing Co.

Huck, P.D., Bateson, C., Volk, R., Cartellier, A., Bourgoin, M. \& Aliseda, A. 2018 The role of collective effects on settling velocity enhancement for inertial particles in turbulence. J. Fluid Mech. 846, 1059-1075.

Hunt, J.C.R., Wray, A.A. \& Moin, P. 1988 Eddies, streams and convergence zones in turbulent flows. In Proceedings of the 1988 Summer Program, Center for Turbulence Research, Stanford, CA, pp. 193-208.

Ireland, P.J., BRagG, A.D. \& Collins, L.R. $2016 a$ The effect of Reynolds number on inertial particle dynamics in isotropic turbulence. Part 1. Simulations without gravitational effects. J. Fluid Mech. 796, 617-658.

Ireland, P.J., Bragg, A.D. \& Collins, L.R. $2016 b$ The effect of Reynolds number on inertial particle dynamics in isotropic turbulence. Part 2. Simulations with gravitational effects. J. Fluid Mech. 796, 659-711.

Jung, J., YeO, K. \& LeE, C. 2008 Behavior of heavy particles in isotropic turbulence. Phys. Rev. E 77, 016307.

Kolmogorov, A.N. 1941 The local structure of turbulence in incompressible viscous fluid for very large Reynolds numbers. Dokl. Akad. Nauk SSSR 30, 299-303.

LiEN, R.-C. \& D'ASARO, E.A. 2002 The kolmogorov constant for the lagrangian velocity spectrum and structure function. Phys. Fluids 14 (12), 4456-4459.

Mathai, V., Calzavarini, E., Brons, J., Sun, C. \& Lohse, D. 2016 Microbubbles and microparticles are not faithful tracers of turbulent acceleration. Phys. Rev. Lett. 117 (2), 024501.

Mathai, V., Huisman, S.G., Sun, C., Lohse, D. \& Bourgoin, M. 2018 Dispersion of air bubbles in isotropic turbulence. Phys. Rev. Lett. 121 (5), 054501.

MAXEY, M.R. 1987 The gravitational settling of aerosol particles in homogeneous turbulence and random flow fields. J. Fluid Mech. 174, 441-465.

MAXEY, M.R. \& RILEY, J.J. 1983 Equation of motion for a small rigid sphere in a nonuniform flow. Phys. Fluids 26 (4), 883.

Mittal, R., Ni, R. \& SeO, J.-H. 2020 The flow physics of covid-19. J. Fluid Mech. 894, F2.

Momenifar, M. \& BRAGG, A.D. 2020 Local analysis of the clustering, velocities, and accelerations of particles settling in turbulence. Phys. Rev. Fluids 5 (3), 034306.

Mordant, N., LÉvêque, E. \& Pinton, J.-F. 2004 Experimental and numerical study of the lagrangian dynamics of high Reynolds turbulence. New J. Phys. 6 (1), 116.

Mordant, N., Metz, P., Michel, O. \& Pinton, J.-F. 2001 Measurement of lagrangian velocity in fully developed turbulence. Phys. Rev. Lett. 87 (21), 214501.

Nemes, A., Dasari, T., Hong, J., Guala, M. \& Coletti, F. 2017 Snowflakes in the atmospheric surface layer: observation of particle-turbulence dynamics. J. Fluid Mech. 814, 592-613.

NiELSEN, P. 1993 Turbulence effects on the settling of suspended particles. SEPM J. Sedim. Res. 63, 835-838.

Ouellette, N.T., Xu, H., Bourgoin, M. \& Bodenschatz, E. 2006 Small-scale anisotropy in Lagrangian turbulence. New J. Phys. 8 (6), 102.

Parishani, H., Ayala, O., Rosa, B., Wang, L.-P. \& GrabowsKi, W.W. 2015 Effects of gravity on the acceleration and pair statistics of inertial particles in homogeneous isotropic turbulence. Phys. Fluids 27 (3), 033304.

Perry, A.E. \& ChOng, M.S. 1994 Topology of flow patterns in vortex motions and turbulence. Appl. Sci. Res. 53 (3-4), 357-374.

Petersen, A.J., BAKer, L. \& Coletti, F. 2019 Experimental study of inertial particles clustering and settling in homogeneous turbulence. J. Fluid Mech. 864, 925-970.

Pope, S.B. 2000 Turbulent Flows. Cambridge University Press. 


\section{Dynamics of small heavy particles in homogeneous turbulence}

Pope, S.B. 2014 The determination of turbulence-model statistics from the velocity-acceleration correlation. J. Fluid Mech. 757, R1.

Pozorski, J. \& Minier, J.-P. 1998 On the lagrangian turbulent dispersion models based on the Langevin equation. Intl J. Multiphase Flow 24 (6), 913-945.

RosA, B. \& POZORSKI, J. 2017 Impact of subgrid fluid turbulence on inertial particles subject to gravity. J. Turbul. 18 (7), 634-652.

Saddoughi, S.G. \& VeEravalli, S.V. 1994 Local isotropy in turbulent boundary layers at high Reynolds number. J. Fluid Mech. 268, 333-372.

SALAZAR, J.P.L.C. \& Collins, L.R. 2012 Inertial particle acceleration statistics in turbulence: effects of filtering, biased sampling, and flow topology. Phys. Fluids 24 (8), 083302.

SAWFORD, B.L. 1991 Reynolds number effects in Lagrangian stochastic models of turbulent dispersion. Phys. Fluids A 3 (6), 1577-1586.

Sawford, B.L., Yeung, P.K., Borgas, M.S., Vedula, P., La Porta, A., Crawford, A.M. \& Bodenschatz, E. 2003 Conditional and unconditional acceleration statistics in turbulence. Phys. Fluids 15 (11), 3478-3489.

SQUiRES, K.D. \& EATON, J.K. 1991a Measurements of particle dispersion obtained from direct numerical simulations of isotropic turbulence. J. Fluid Mech. 226, 1-35.

SQuires, K.D. \& EATON, J.K. 1991 breferential concentration of particles by turbulence. Phys. Fluids A 3 (5), 1169-1178.

Sreenivasan, K.R. 1998 An update on the energy dissipation rate in isotropic turbulence. Phys. Fluids 10 (2), 528-529.

TCHEN, C.M. 1947 Mean value and correlation problems connected with the motion of small particles suspended in a turbulent fluid. PhD thesis, TU Delft.

TENNEKES, H. \& LUMLEY, J.L. 1972 A first course in turbulence. MIT press.

TOM, J. \& BRAGG, A.D. 2019 Multiscale preferential sweeping of particles settling in turbulence. J. Fluid Mech. 871, 244-270.

VARIANO, E.A. \& COWEN, E.A. 2008 A random-jet-stirred turbulence tank. J. Fluid Mech. 604, 1-32.

VASsilicos, J.C. 2015 Dissipation in turbulent flows. Annu. Rev. Fluid Mech. 47, 95-114.

Volk, R., Calzavarini, E., Verhille, G., Lohse, D., Mordant, N., Pinton, J.-F. \& Toschi, F. 2008 Acceleration of heavy and light particles in turbulence: comparison between experiments and direct numerical simulations. Physica D 237 (14-17), 2084-2089.

Voth, G.A., La Porta, A., Crawford, A.M., Alexander, J. \& Bodenshatz, E. 2002 Fully resolved simulations of particle-turbulence interaction. J. Fluid Mech. 469, 121-160.

WANG, L.-P. \& MAXEY, M.R. 1993 Settling velocity and concentration distribution of heavy particles in homogeneous isotropic turbulence. J. Fluid Mech. 256, 27-68.

WANG, L.-P. \& Stock, D.E. 1993 Dispersion of heavy particles by turbulent motion. J. Atmos. Sci. 50 (13), 1897-1913.

Wilkinson, M. \& Mehlig, B. 2005 Caustics in turbulent aerosols. Europhys. Lett. 71 (2), $186-192$.

Worth, N.A. \& Nickels, T.B. 2011 Some characteristics of thin shear layers in homogeneous turbulent flow. Phil. Trans. R. Soc. A 369 (1937), 709-722.

Worth, N.A., Nickels, T.B. \& Swaminathan, N. 2010 A tomographic PIV resolution study based on homogeneous isotropic turbulence DNS data. Exp. Fluids 49 (3), 637-656.

YANG, T.S. \& SHY, S.S. 2005 Two-way interaction between solid particles and homogeneous air turbulence: particle settling rate and turbulence modification measurements. J. Fluid Mech. 526, 171-216.

YEUNG, P.K. 2001 Lagrangian characteristics of turbulence and scalar transport in direct numerical simulations. J. Fluid Mech. 427, 241-274.

Yeung, P.K., Donzis, D.A. \& SReEnivasan, K.R. 2012 Dissipation, enstrophy and pressure statistics in turbulence simulations at high Reynolds numbers. J. Fluid Mech. 700, 5-15.

Yosнiмото, H. \& Gото, S. 2007 Self-similar clustering of inertial particles in homogeneous turbulence. J. Fluid Mech. 577, 275-286.

Yudine, M.I. 1959 Physical considerations on heavy-particle diffusion. Adv. Geophys. 6, 185-191.

Zaichik, L.I., Simonin, O. \& Alipchenkov, V.M. 2003 Two statistical models for predicting collision rates of inertial particles in homogeneous isotropic turbulence. Phys. Fluids 15 (10), 2995-3005.

ZAmAnsky, R., Vinkovic, I. \& GoROKhovski, M. 2013 Acceleration in turbulent channel flow: universalities in statistics, subgrid stochastic models and an application. J. Fluid Mech. 721, 627-668.

ZhANG, Z., LEGENDRE, D. \& ZAMANSKY, R. 2019 Model for the dynamics of micro-bubbles in high-Reynolds-number flows. J. Fluid Mech. 879, 554-578. 